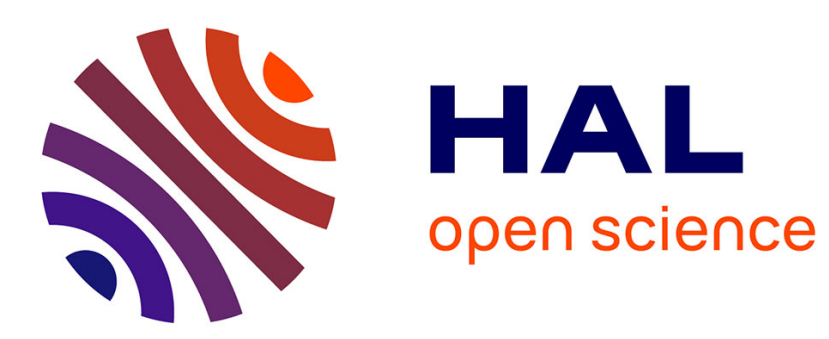

\title{
Traits morphologiques originaux du plateau de Montredon- Labessonnié (Sud-Ouest du Massif Central)
}

Georges Bertrand

\section{To cite this version:}

Georges Bertrand. Traits morphologiques originaux du plateau de Montredon- Labessonnié (SudOuest du Massif Central). Revue Géographique des Pyrénées et du Sud-Ouest, 1960, 31 (3), pp.277293. 10.3406/rgpso.1960.4730 . hal-02570345

\section{HAL Id: hal-02570345 \\ https://hal.science/hal-02570345}

Submitted on 12 May 2020

HAL is a multi-disciplinary open access archive for the deposit and dissemination of scientific research documents, whether they are published or not. The documents may come from teaching and research institutions in France or abroad, or from public or private research centers.
L'archive ouverte pluridisciplinaire HAL, est destinée au dépôt et à la diffusion de documents scientifiques de niveau recherche, publiés ou non, émanant des établissements d'enseignement et de recherche français ou étrangers, des laboratoires publics ou privés. 


\section{Traits morphologiques originaux du plateau de Montredon- Labessonnié (Sud-Ouest du Massif Central)}

\section{Georges Bertrand}

\section{Résumé}

Le plateau correspond à la surface éogène qui s'est conservée en position déprimée jusqu'à la fin du Tertiaire. Ensuite, la modification du climat, accompagnée d'un soulèvement tectonique, a entraîné le creusement de gorges (Agout, Dadou) à l'aspect « inachevé ». Les affleurements massifs de quartz se hérissent de buttes tout en constituant une zone de convergence hydrographique. Les rivières, respectant la roche massive ; se sont facilement glissées dans les réseaux de diaclases sans cependant parvenir à aménager les versants qui sont calqués sur la structure.

\section{Citer ce document / Cite this document :}

Bertrand Georges. Traits morphologiques originaux du plateau de Montredon-Labessonnié (Sud-Ouest du Massif Central). In: Revue géographique des Pyrénées et du Sud-Ouest, tome 31, fascicule 3, 1960. pp. 277-293;

doi : https://doi.org/10.3406/rgpso.1960.4730

https://www.persee.fr/doc/rgpso_0035-3221_1960_num_31_3_4730

Fichier pdf généré le 05/04/2018 


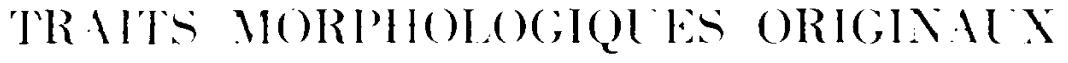

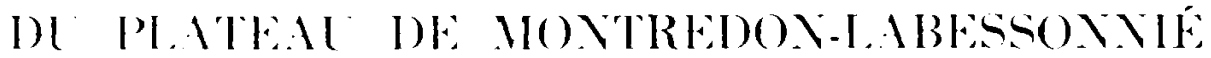 (SLI)-()LEST DI MISAIF CENTRAL) \\ par Georges BFITTAND}

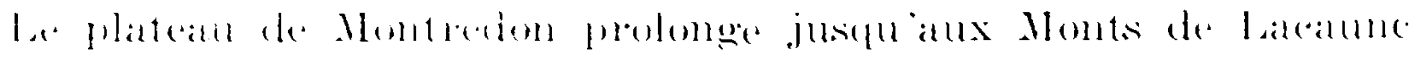

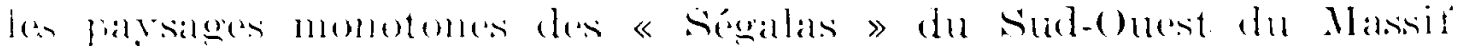

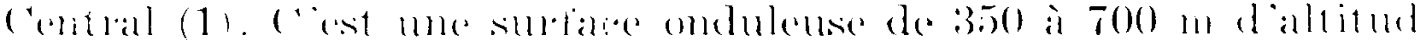

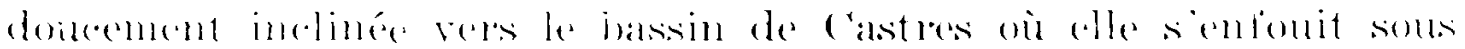

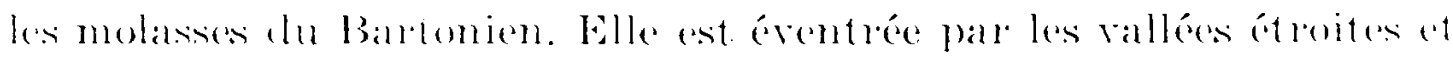

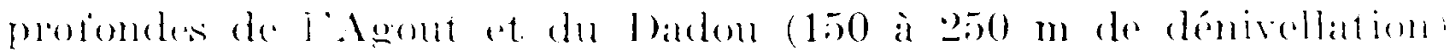
(tix. 1 (1)

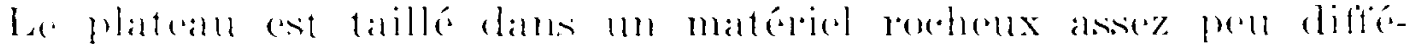

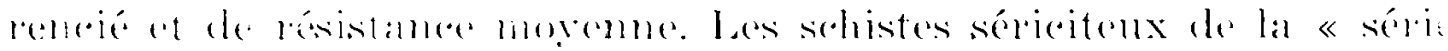

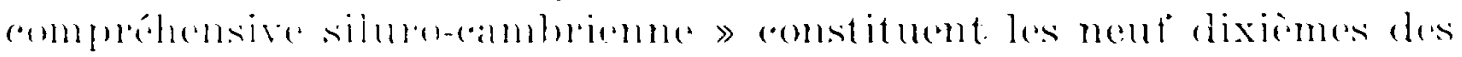
afflumements. ('

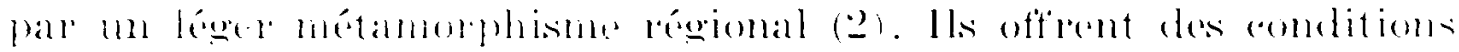

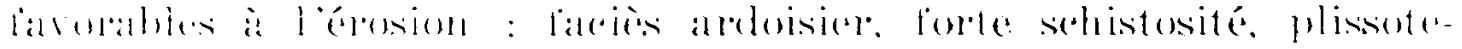

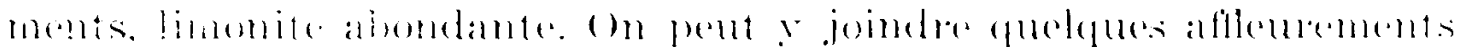

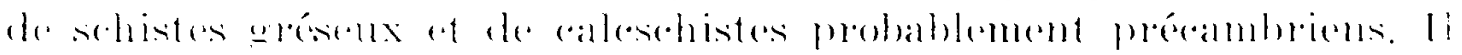

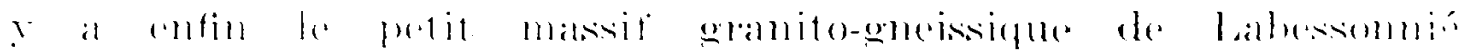

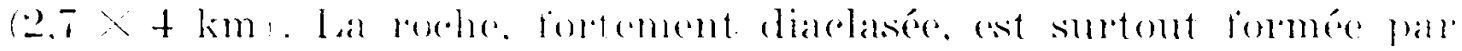

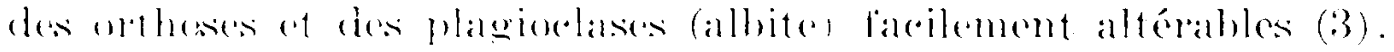

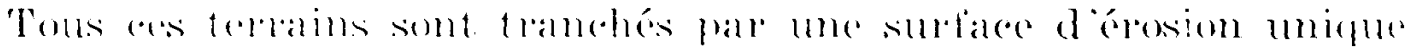

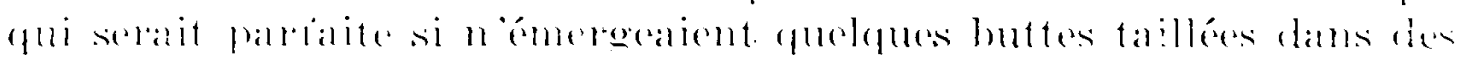

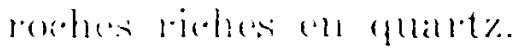

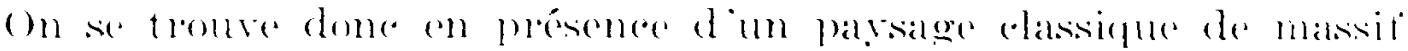

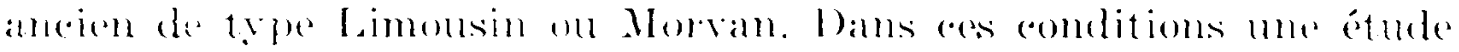
densemble ne présenterait gruère d’intérêt. Par eontre. on doit somb-

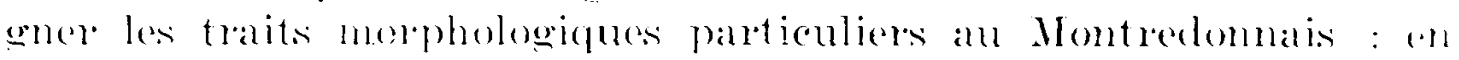

(1) Cotte region n'a jamais fait lobjet d'ume etude morphologique d'ensemble.

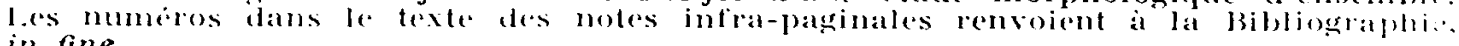
in fine.

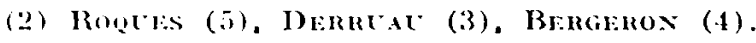

(3) Benglion (4). 


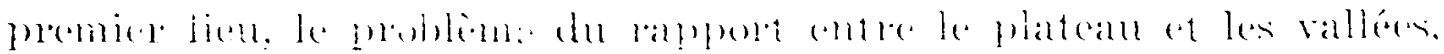

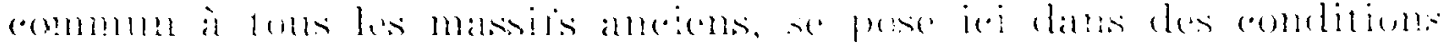

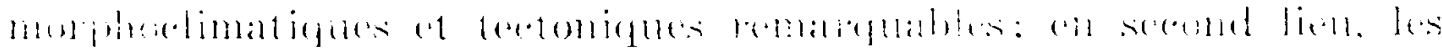

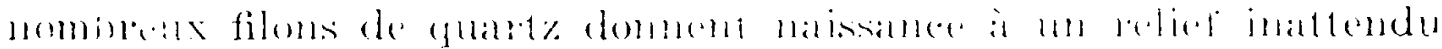

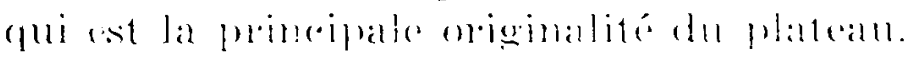

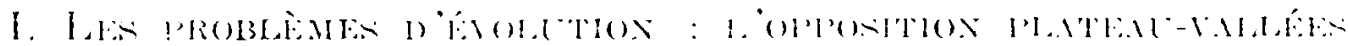

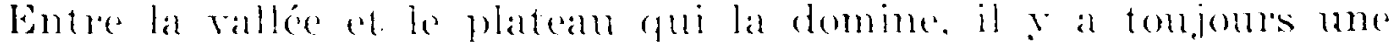

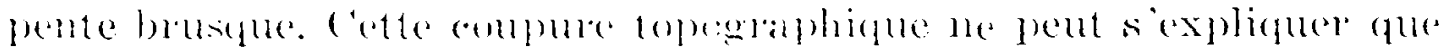

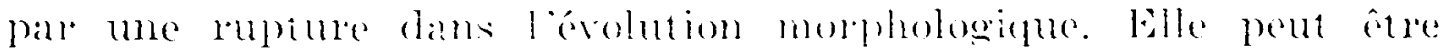

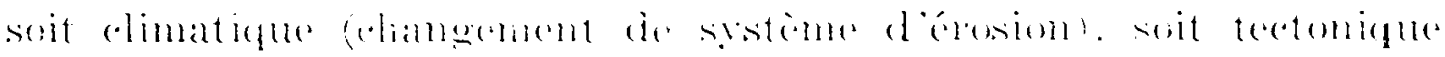

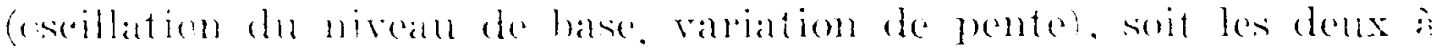

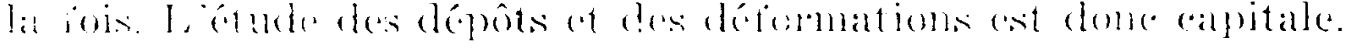

1. Signification morpholegique des argiles rouges à graviers. -...

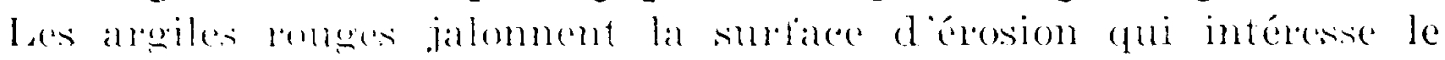

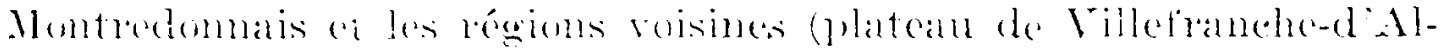

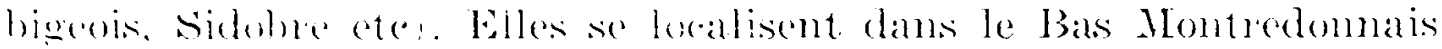

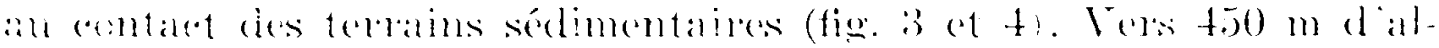
titule clles se terminent en hisean par une linite dérosion. Le dépot

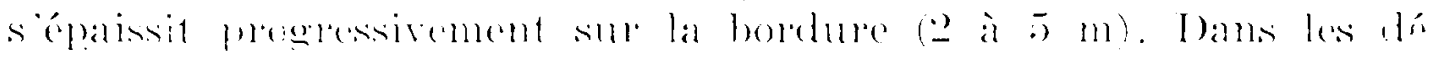

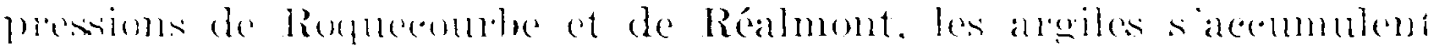
sur 50 is $80 \mathrm{~m}$ d'epaisseur.

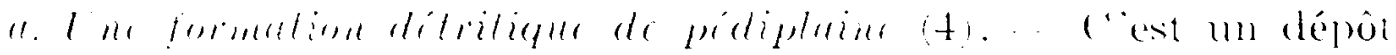

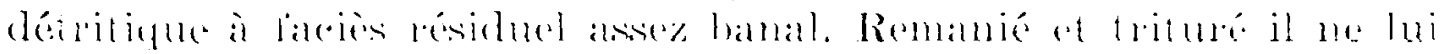

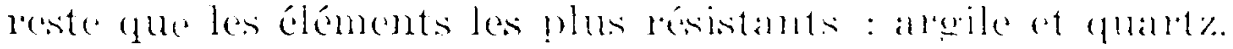

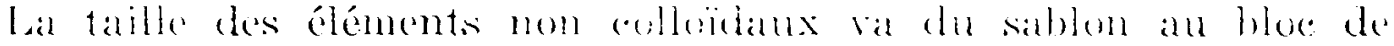

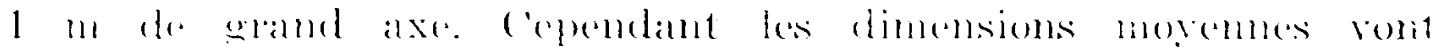
de 2 a l.

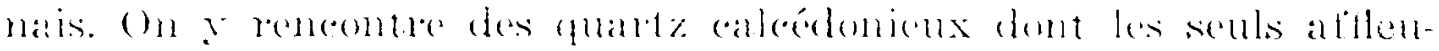

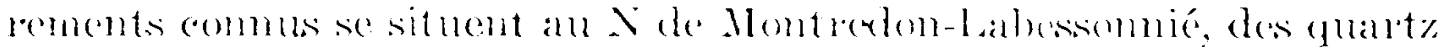

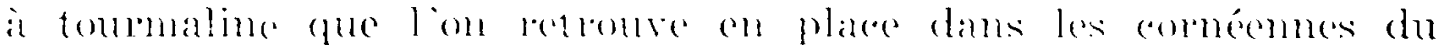

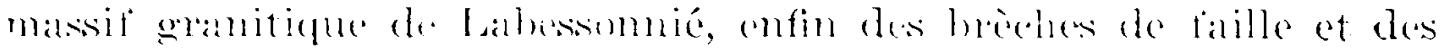
farements dhematile qui abundent sur lont le platealu.

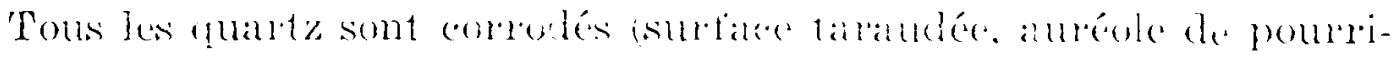

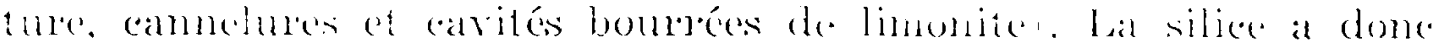

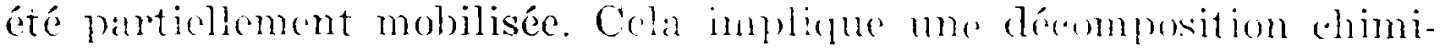

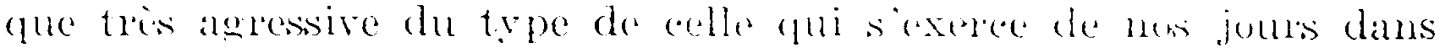
la rone tropicale humide (5).

(9) Mrinato (10), Ravilue de Fortaxilir (11).

(5) Tricarr et Callecx, Introduction a la morphologie climalique. Paris, C. D. Li. 
PLATEAU DE MONTREDON-LABESSONNIE

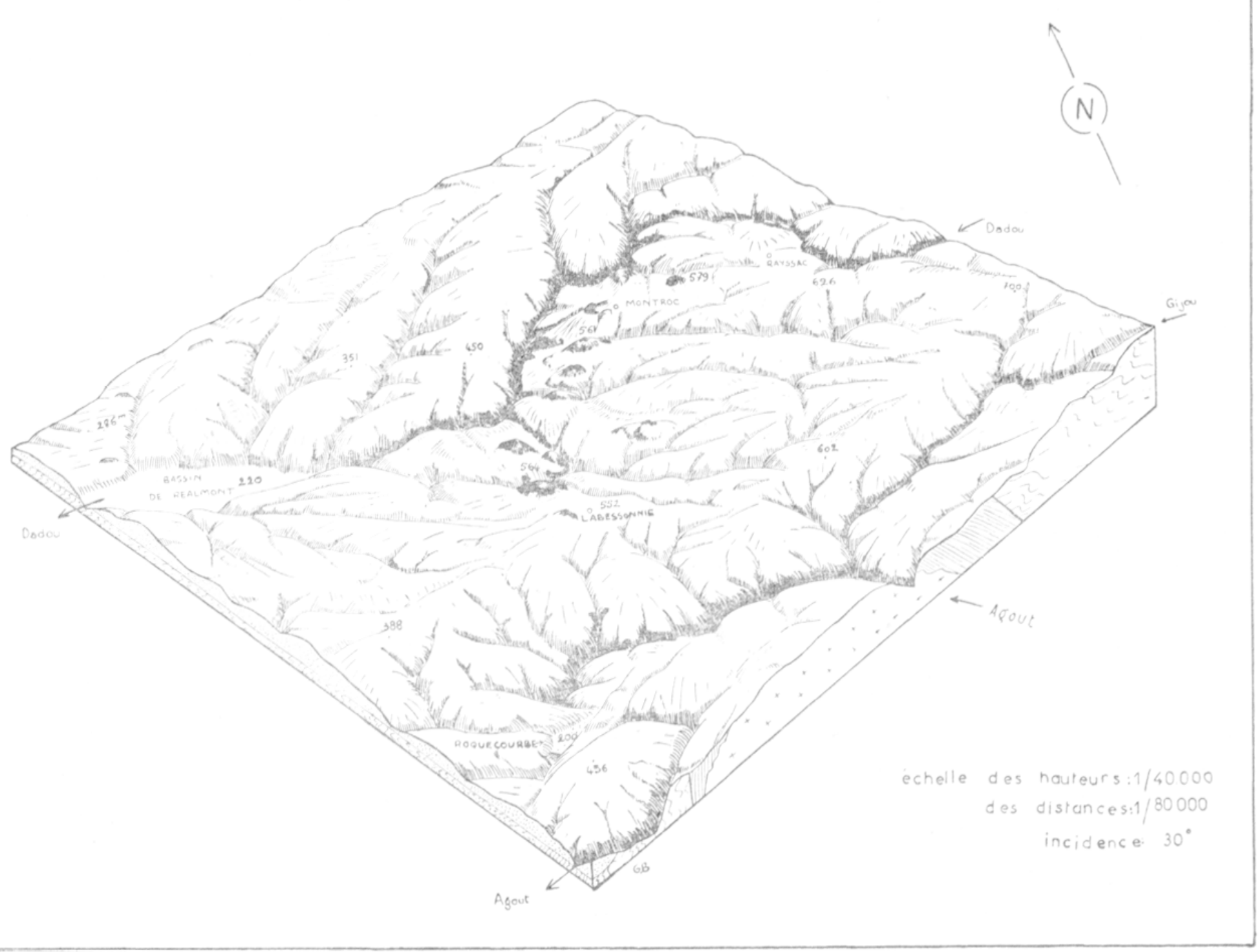

Fifi. 1

I. platrau de Montredon-Iabessonnié. 


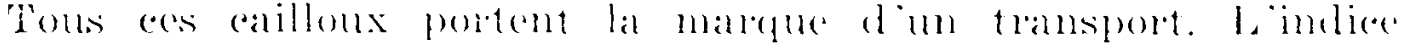

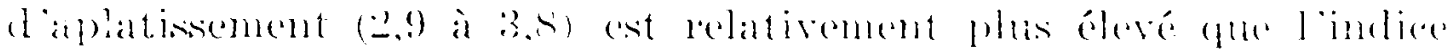

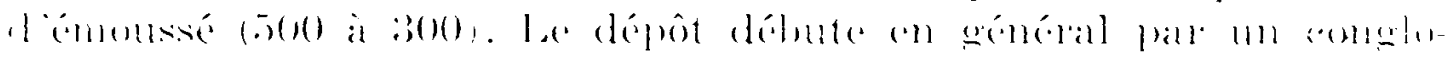
méal de hase ravinam les sohistes.

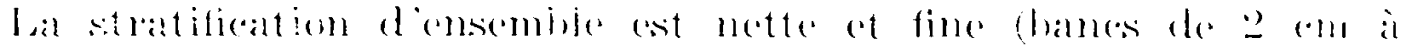

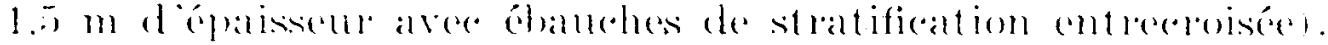

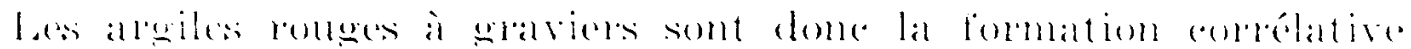

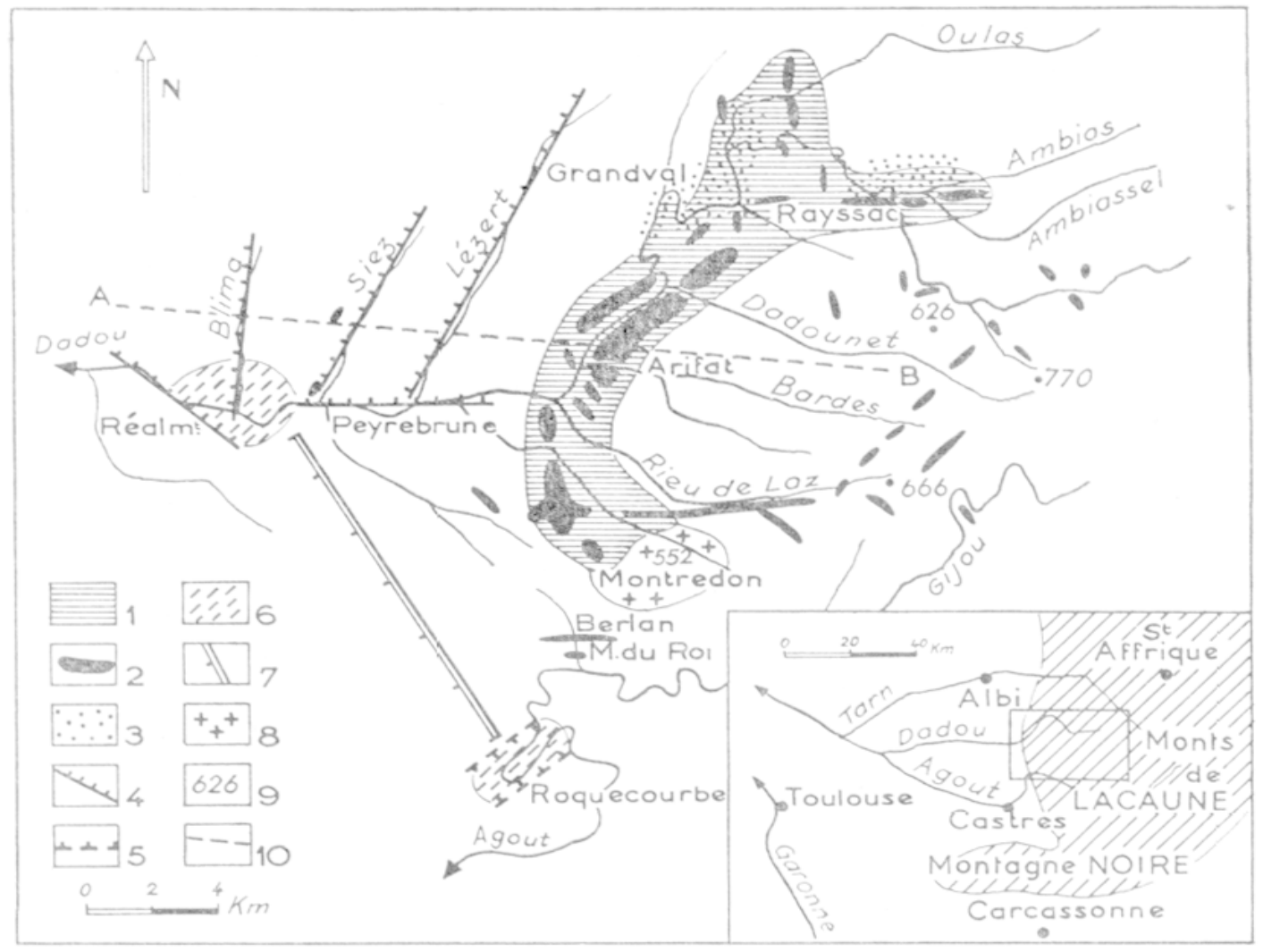

Fili. 2

Tectoniqu: et réseau hydrographique du plateau nontredonnais.

1. Muratle de quartz. … 2. Cirands filons. 3. Mylonitisation. 4. Faille s. Fatle probable. b. Bassin deffomdrement. T. Flestire marginale. 8. Massif franite-gneissique. 9. Nltitude en metre.

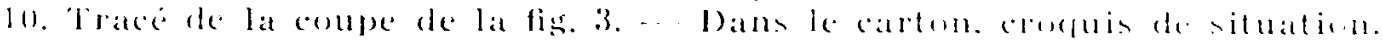

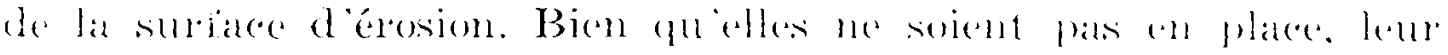

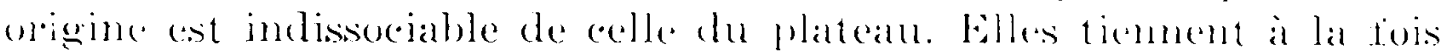

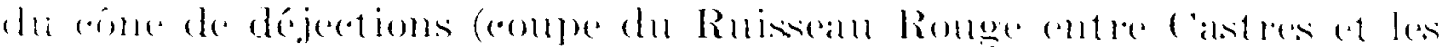

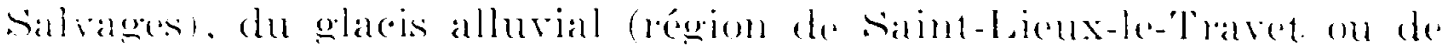

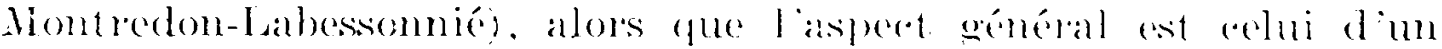
piemont conbryomaire. Cette acelmulation correspond an terme déxolution de la surfare (finessere de la stratifiation at des débriss. 


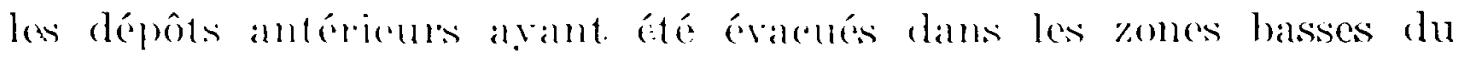
Bassin d Aquitaine (6).

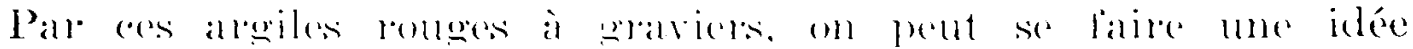

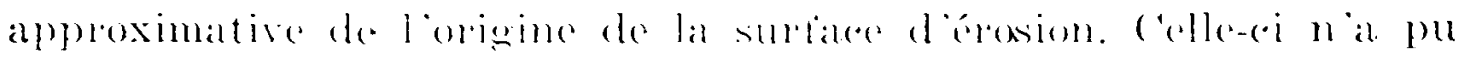

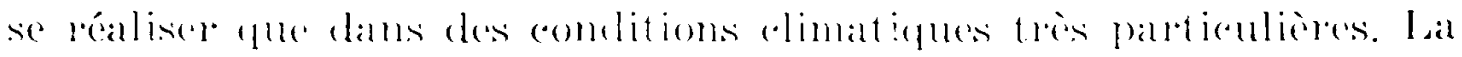
violence de lattaque phesies-ehimigue (mobilisation des silieates, forte proportion des ablobles of la formation de mappes alluriales

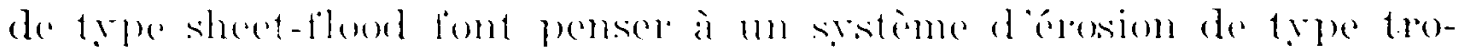

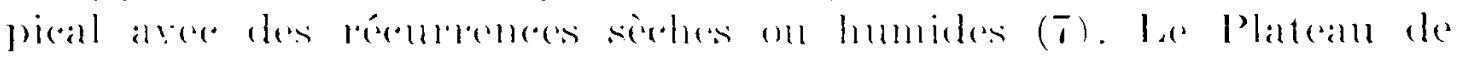
Mentredon correspondrate à ane pédiplatine presque parlate sur-

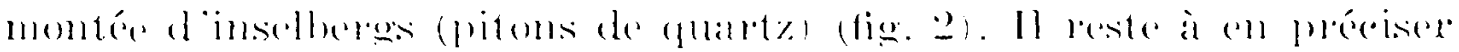
l'âge.

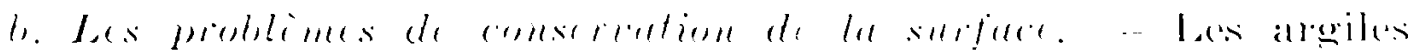

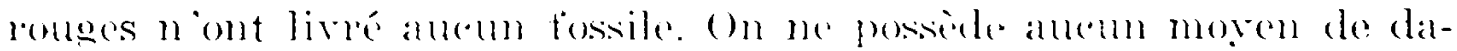

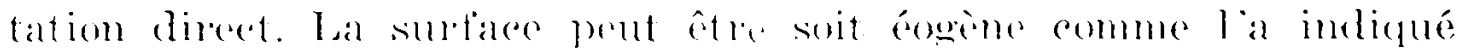

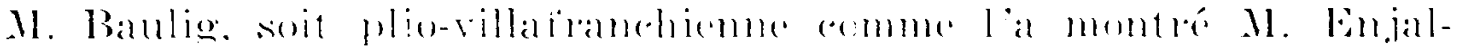
bert (s) a propos des platealux du Viaur. Le senl moyen de datation

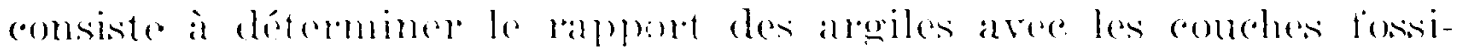
liferes du basion de castres.

Les argiles rouges sont ume bormation détritigue de piemont rem-

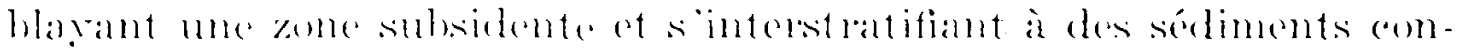

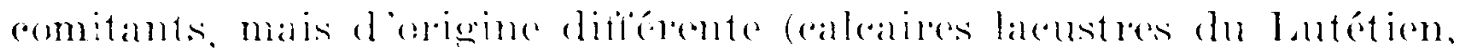

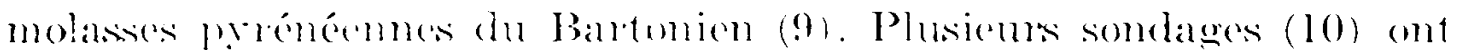

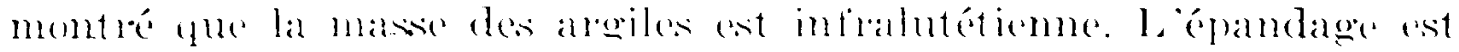

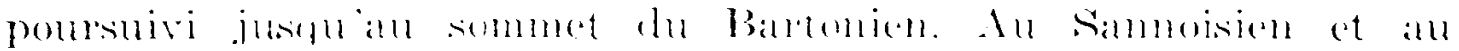

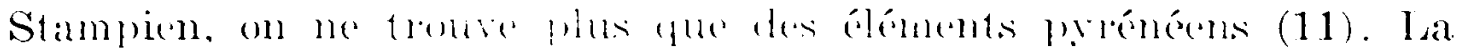

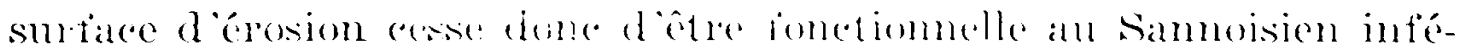

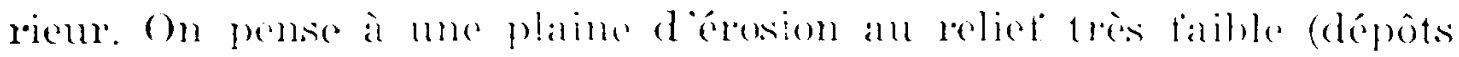

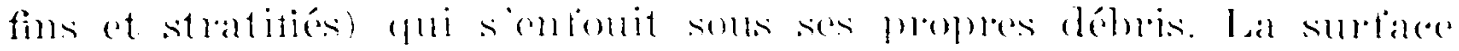

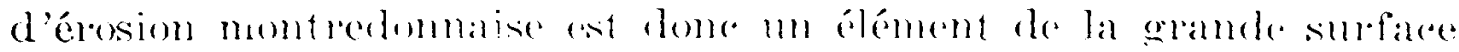
éogine (12).

(i) $M 1 \mathrm{x}(\mathrm{int}) \quad(9)$.

(7) Ces fatts sont corrobores par les fossiles decouverts dams le rertiaire du

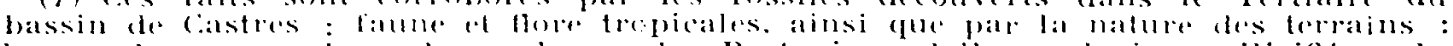

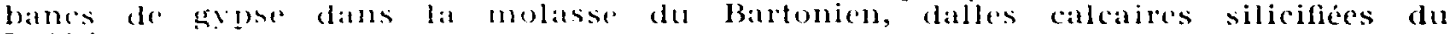
I.utetion.

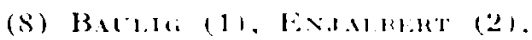

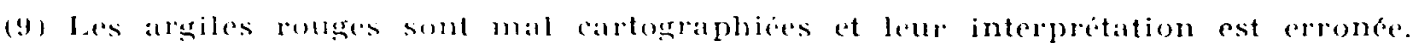

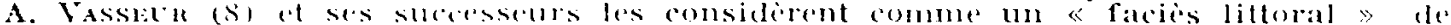

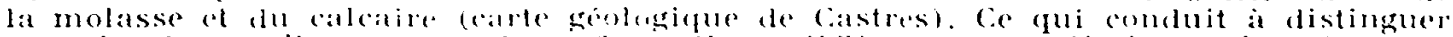
au sein des argiles rouses deux fornations dinerentes aux limites arbitraires.

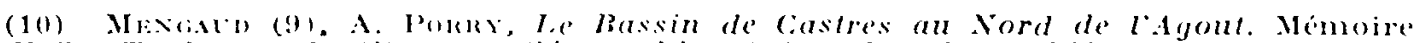
I). E. S., 'Toulouse, Institut de" ciengaphie, 1910 (alactylographic).

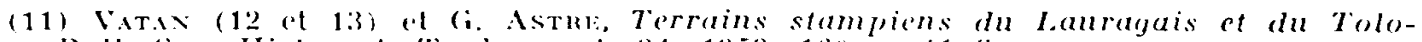

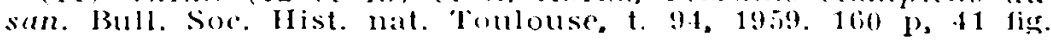

(12) $13 \times$ CLIG (1). 
Cette origine très ancienne pose un probleme d'évolution fondamental. Comment un reliet aequis dès de nilieu du Tertiaire dans un materiel de résistance morenne a-t-il pu être conservé dans un remalefuable étal de frâteheme? Le tait est d'autant plus extraordiniame que le revetement argileux fragile (il n'est consoliclé pan. aucun ciment) $n$ a été que très particllement déapé et reste perché a plus de $200 \mathrm{~m}$ au-dessus des vallées. Seul un mourement tectonigue très réent peut dissiper ce paradoxe : conservation d'une vieille surtace et entoncement brutal des vallées.

2. Possibilité d'un mouvement tectonique récent. - u. T'cctonique et cnfoncement dos cours d'eau. - le creusement des vallées n'a pu s'eflectuer qu à la laveur d'un changement du sistème dérosion au Quaternaire. La coneentration et labondance des eaux eou. rantes. l'atetion du gel et la cryoturbation peurent rendre compte de lobaboration des vallées. Mais elles ne suffisent pas. Il falli alusi que les conditions de pente soient farorables.

Lia surlace a ité déformée postérieurement an dépôt des argiles rouges. Plusiours observations comeordantes laissent penser qu "il s’agit d’une déformation très réecente. 1)’une part, il s'est écoulé entre le Bartonien ot le Quaternaire 11 long espace de temps sams que la surface égène ait été sensiblement modifiée. On ne pent pas invonuer whe fossilisation sous le calcaire on la molasse. I a surfiace n a done pu se conserver qu èn position déprimée sous une mince converture d argiles rouges. I) autre part, comme nous le verrons plus loin, les vallées présentent des formes « récentes » (versints raides et peu evolués. ruptures de pente du profil en long). Enfin. il faut coneore insister sur la position culminante des arowiles rouges. Elles sont tranchées à vif par les vallées ot conservées en inversion de relief meme sur les extrémités des interflures. Souvent la formation n'a pas été dérangée et eonserve, au sommet diun versant, la stralification acquise au Tertiaire.

Na-t-on pas là un relief récent directement lie à un soulerement? 1. hy pothèse d im mobilisme quaternaire n est peut-otre pas à exelure. C'ost le seul moren logique d expliquer le contraste brutal antre le piatean ot los vallées, bien que rien ne laisse supposer que les vallées soient synorogeniques.

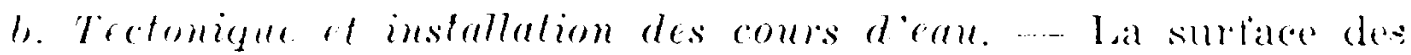
argiles rouges a subi un gauchissement d'ensemble gui l'a portée aux altitudes actuelles, cest-à-dire à plusieurs centaines de mètres au-clessus du niveau qu'elle devait oceuper au Tertiaire. Le sondolement. s'est effectué a partir d'une flexure marginale allant de 


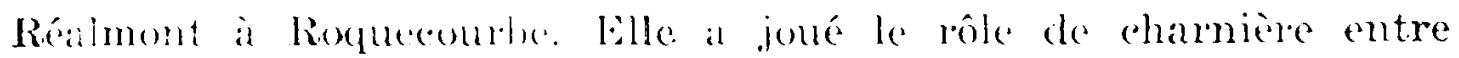
le bissin de ciastres sulsident ot le Montrelonnais en voie de sould-

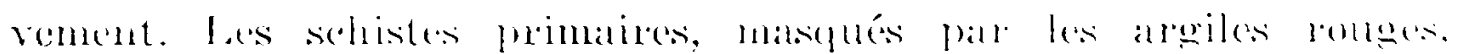

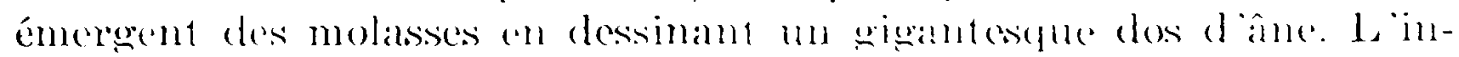
tensité du plongennent valie de Réalmont où il est taible. à Roquecourbe où il passe a la laille flexurée.

la bordule est compliqué par deux bassins tectoniques. A Réalmont se creuse un petit hassin carbonitione et permien qui a rejoué au Tertiaire et qui a été partiellement eomblé d'argiles romges. La

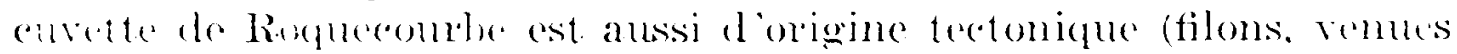

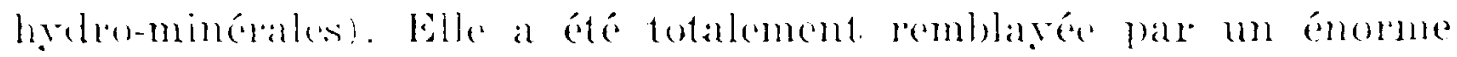

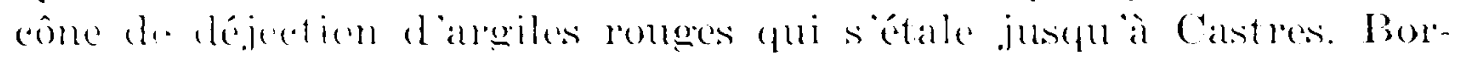
dure mise a part, il ny a pas en. depuis l'etablisisement de lat sur-

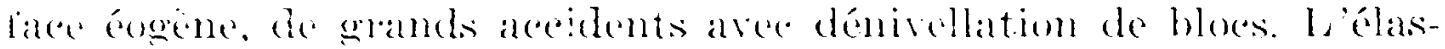

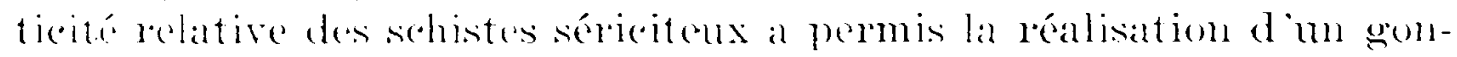

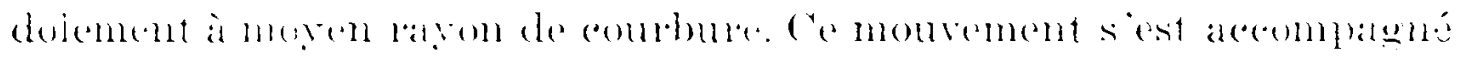

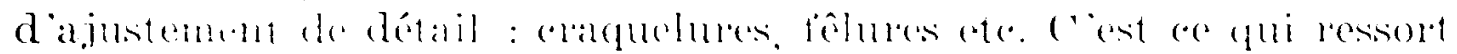
de lexamen des filons de quartz dinns les galeries de mines. Les miroirs de fillle sont rares at les décrochements de fabbe amplitude $(1: 3)$.

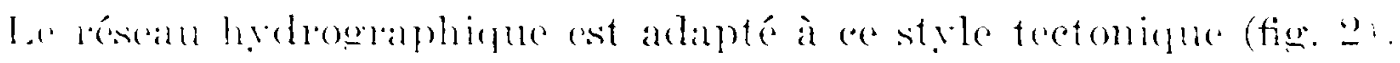
las antires maldessess sont comsépuentes à la pente générale de la

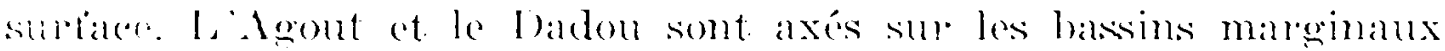
de Roguecourbe et Réahmont qui ont servi de niveaux de bise ì

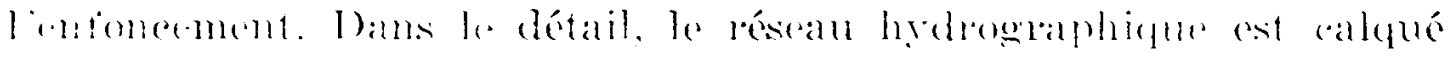

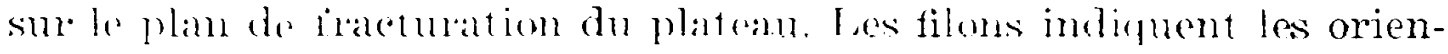

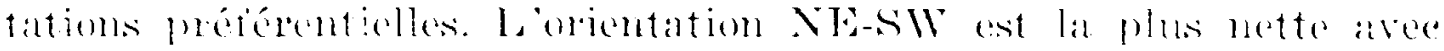

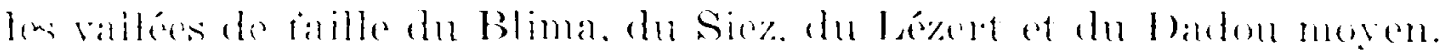

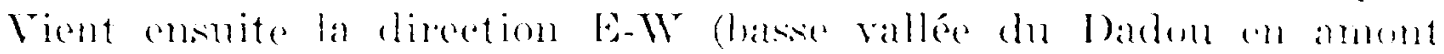

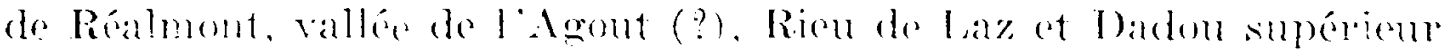

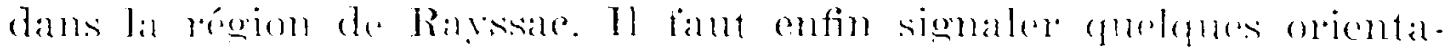

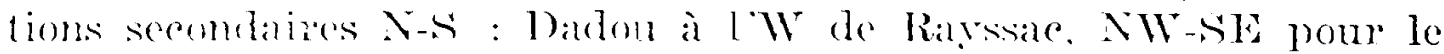
barkme en amont de Salint-olean-drobanes.

Las rivienes milisent des lignes de broyage (6ulas, Dadou dans la

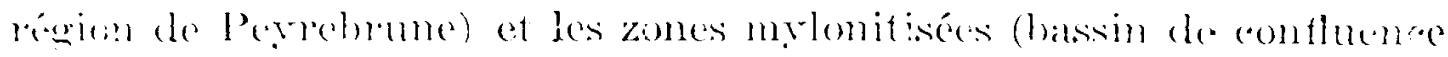

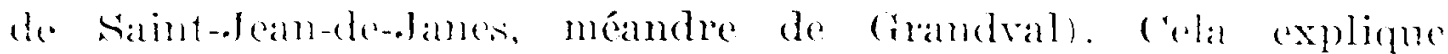

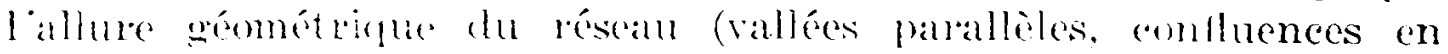
angle droit) et acentuteneor. l’impression générale d inatrèvement.

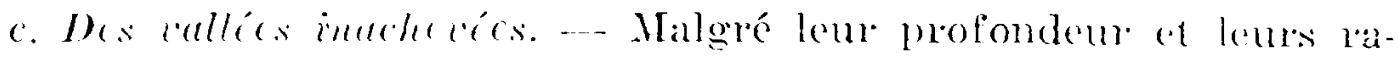
mifications, les vallés présentent un reliet assez peu érolué. láaspect

(13) lacidit: (t) et observations personnelles. 
de crorge domine. Ies versants conservent des pentes raides même dans les schistes tendres de Roquecourbe. Les sols de versant sont minces et discontinus. Dans les prandes vallées tout indique un retald du modelé des versants sur le comsement linéaire. Le contanet entre le plateau et le haut du versant soffectue par une hrustue mupture de pente qui aceentue eneore lopposition plateau-vallé (fig. 4). Seuls les cours d ean tres bien alimentés eommo l' Agont et le Dadou ont pu creuser de profondes rallées. Ies rivières afluluen-

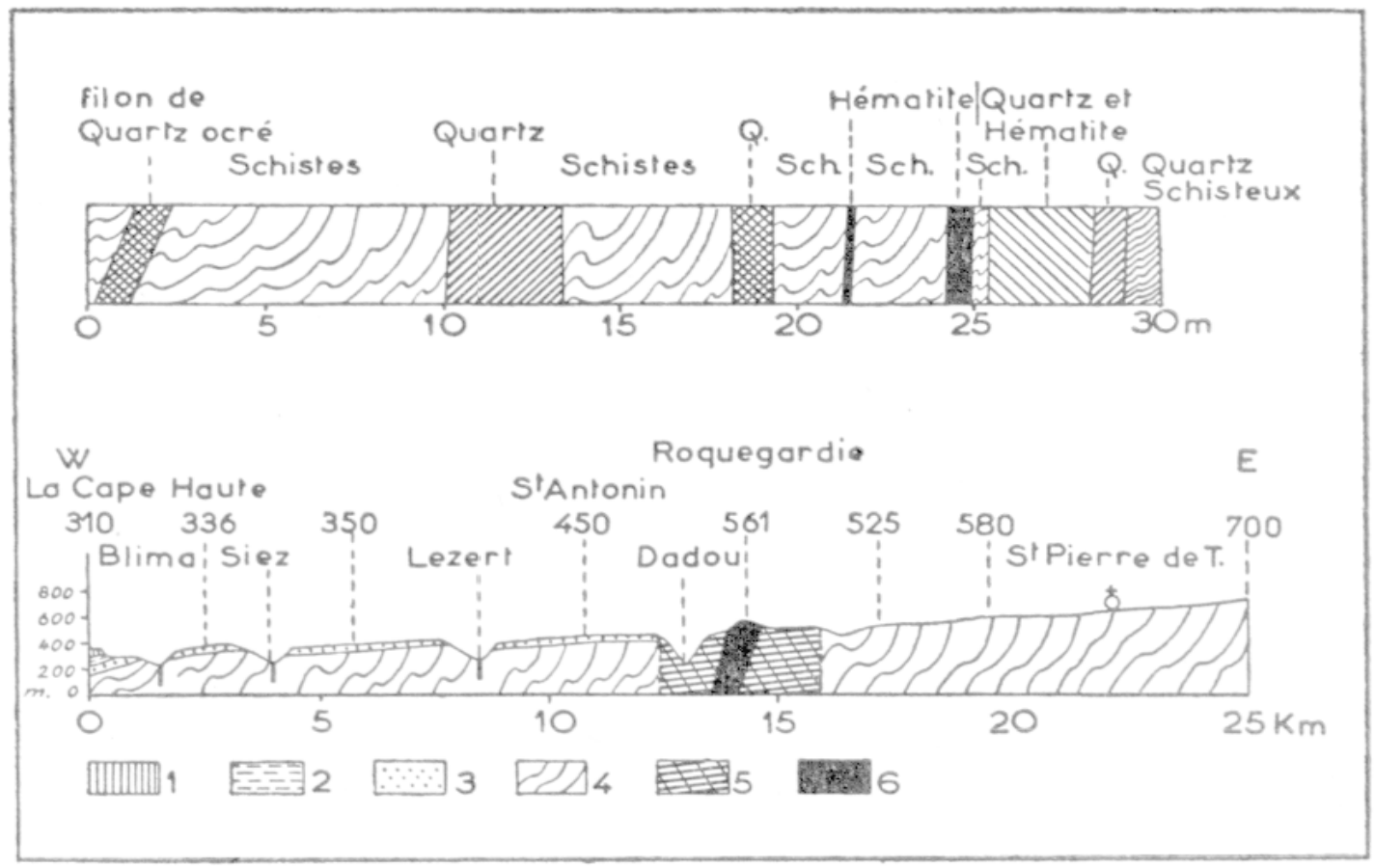

Fig. 3

La muraille de quartz.

En haut, coupe a travers un secteur de la muraille de quart $\%$ (salerie de mine de Montroc). En bas, eoupe selon AB sur la fig. 2 : 1. Calcaire sannoisien. -.. 2. Molasse hartonienne. - .. 3. Argiles pouges a sraviers.

- 4. Schistes siluro-cambriens. - 5. Zone silicifiec. . - G. Quartz.

tes sont restées comme suspendues au-dessus des grandes artères. Leurs vallées n ont pas encore été touchées par la vague d'órosion régressive. Ce sont des vallées « intantiles », à fond malrécalecens. où le modelé des versants a été plus actif que l'enfoncoment. vertical.

Le tracé des vallées est très peu aménagé. Les méandres sont lailres. ()n ne trouve que denx trains de méandres, l'un en amont de Requccourbe, l'autre entre Rayssac et Saint--Jean-de-Janes. Labsence de calibrage paraît due moins à la dureté des roches qu'a la rapidité 
de lenfoncement. la prépondérance de lenfoncement apparaît très netfemont dans le cas des deux méandres abandommés de Travanet qui sont perohés ì differents niveaux au-dessus de la gorere actuelle (fie. 5). ()n pent expliquer de la meme mantè la capture, en fonetion d une zone de mylonites, de la basse vallée de l'ambias par le badour mirux alimenté (fig. 4).

Rien n̈indique que le areusement se soit effectue par étapes. Les replats somt rares ot il n'est pas possible de les raceorder. Le profil en long présente de nombreases irrégularités dorigine structurale (barres de quartz), ce qui interdit toute reconstitution cyclique. Lets

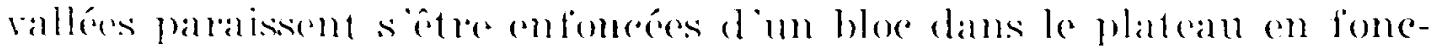
tion de la pente erée par la tectonique.

3. Le modelé périglaciare. -. I đanégald lépartition des phénomènes périchaciatires eontribue eneore a opposer les vallées et le plateau. I)s éfudes réerntes ont fortement insiste sur lubiquite des formes aromivales dams le sud-()uest du Massit Central (14).

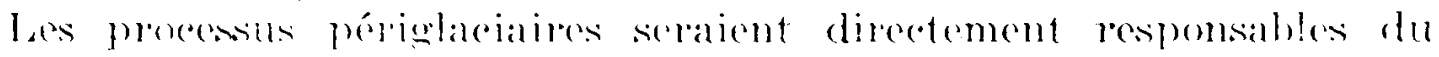

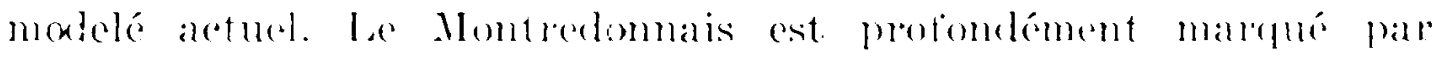
l'aetion du gel et de la solifluetion. Cependant il faut souliener les limites de ces manifistations.

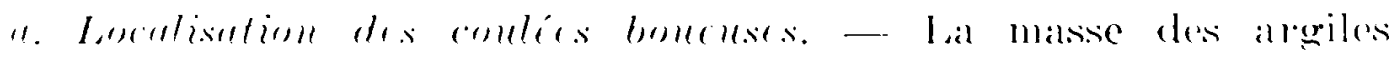
rouges à gravios est dementee en place. Elle conserve presque intaret soil facies de lépot semi-aride. L hypothese de grandes nappes de solifluction remaniant les areiles a maviers et les entrainant dans les zones basses est a rejeter. Pourtant, les areiles constituent un matérel facilement mohilisable. La partic fine est abondante et la pente de la surface forte. Cette immunité pose un problème. On bien les proenssus périglaciaires ont été très peu efficaces ou bien la pente du platean est une acquisition très récente. La deuxième hypothèse est. plus séduisante. car elle corrobore des faits déjà observés (15).

la solifluetion joue. cependant, un rôle important; mais il sagit lomionus de modifications de détail limitées à des points singuliers. Tris peu actif's sur le plateau. les écoulements boucux sontensifien. dans les vallées. Ies moilleures conditions sont rémies dans les petites vallées et dans les « combes ». On y trouve les trois éléments indispensables : un matériel argileux abondant, l'eau qui sourd des nalppes phréatiques et enfin une pente assez forte. On $y$ rencontre de larges coulées de 2 à $6 \mathrm{~m}$ d'épaisseur reconnaissables, cn coupe.

(14) BAFCKEHOOT $(14,15,16)$.

(15) Voir plus haut $\$ 2$, A. 
a leurs guirlandes of rosaces de pierre. Ias whisements sont si importants que le fond des vallons est merowere of lentomerment li-

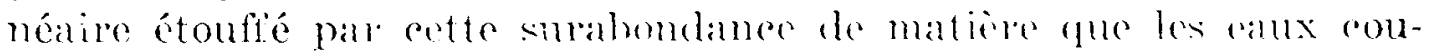

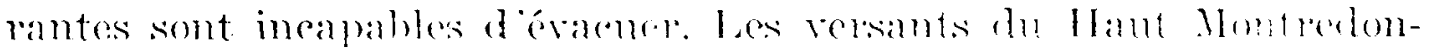

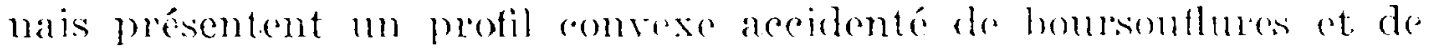
lompes calaretéristiques d'un gelissement en masiot.

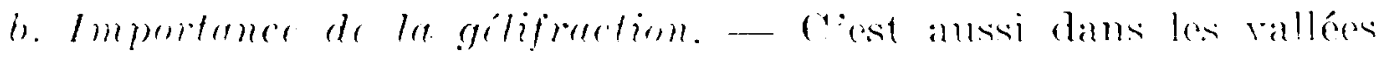
ghe se manifieste le get. Ides sohistes du platean sont protexes par les sols of par les placages d al poiles rouges. Par contre la roche ap-

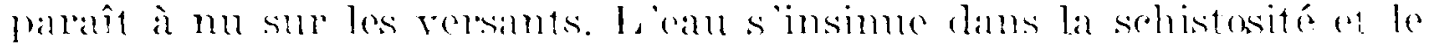

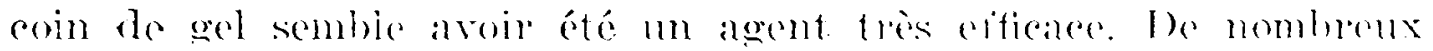

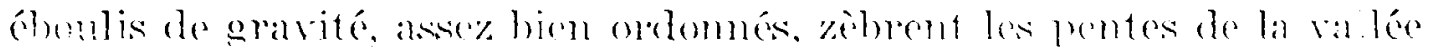

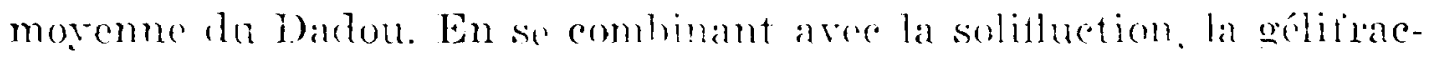
tion domm des grezes de playuettes de sohistes à structure open-work.

I.es formes pépiglaciaires sont superficielles ot foralisés. 1) ailleurs.

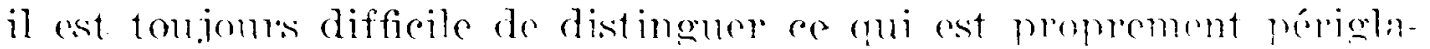

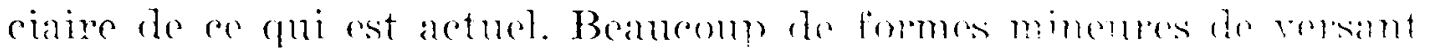

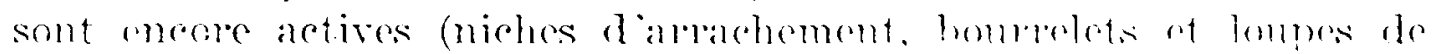
gi isismont).

Le modele cryonival doit être comsidere comme un épiphénomène

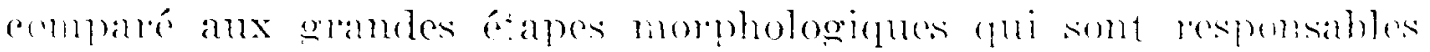

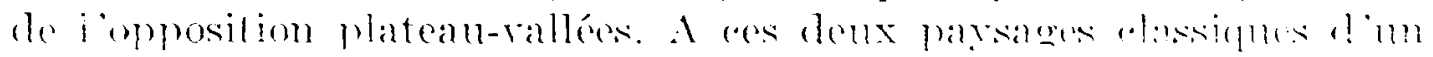

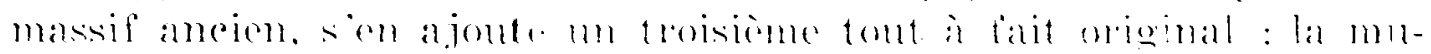
rable de quartz.

\section{IT. IA MIRAILIE INE QTHRT}

les huttes rhantiques. les ressants raides ot mus tailies dans le quarty introduisent des traits montagnards dans me region au reliaf "alme (fig. 1 et 2 : . Le quartz atflenre entre Tabessomie et Paulin stu 2 à $3 \mathrm{~km}$ do large. Il constitue un ensemble homogone et orioinal qui isole le Bas Montredomais du ITant IIontredomais ot que nous appellerons la « muraille de quartz ». Cost lo seul relief struetural du plateau. Il parait reposer sur une contradiction morphologique.

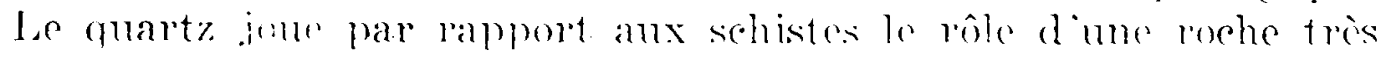
dure eomme le montrent les goreses on trait de seie of les pitome

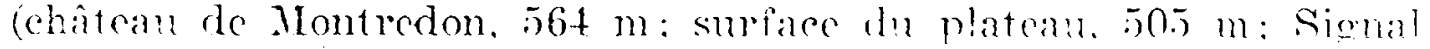
de Mouffe. $576 \mathrm{~m}$; plateau. $520 \mathrm{~m}$ ).

Paradoxalement. le I)adou est adapté aux affeurements quartzeux Il suit toutes les inflexions des harres de quartz (F-IV cntre SaintJean-de-Janes et Rayssac. N-S de Rayssac à la confluenee du Rieu de $[$ Laz). 
iat muraille de quarty est aussi une zone de comvergenes hroleoeraphigue. Les afluents de rive gauche du Dadou viement y rom-

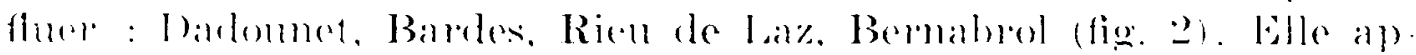

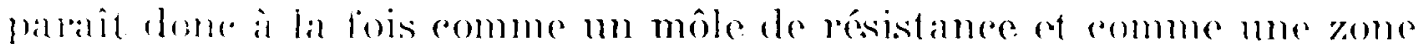
d. !aiblesse.

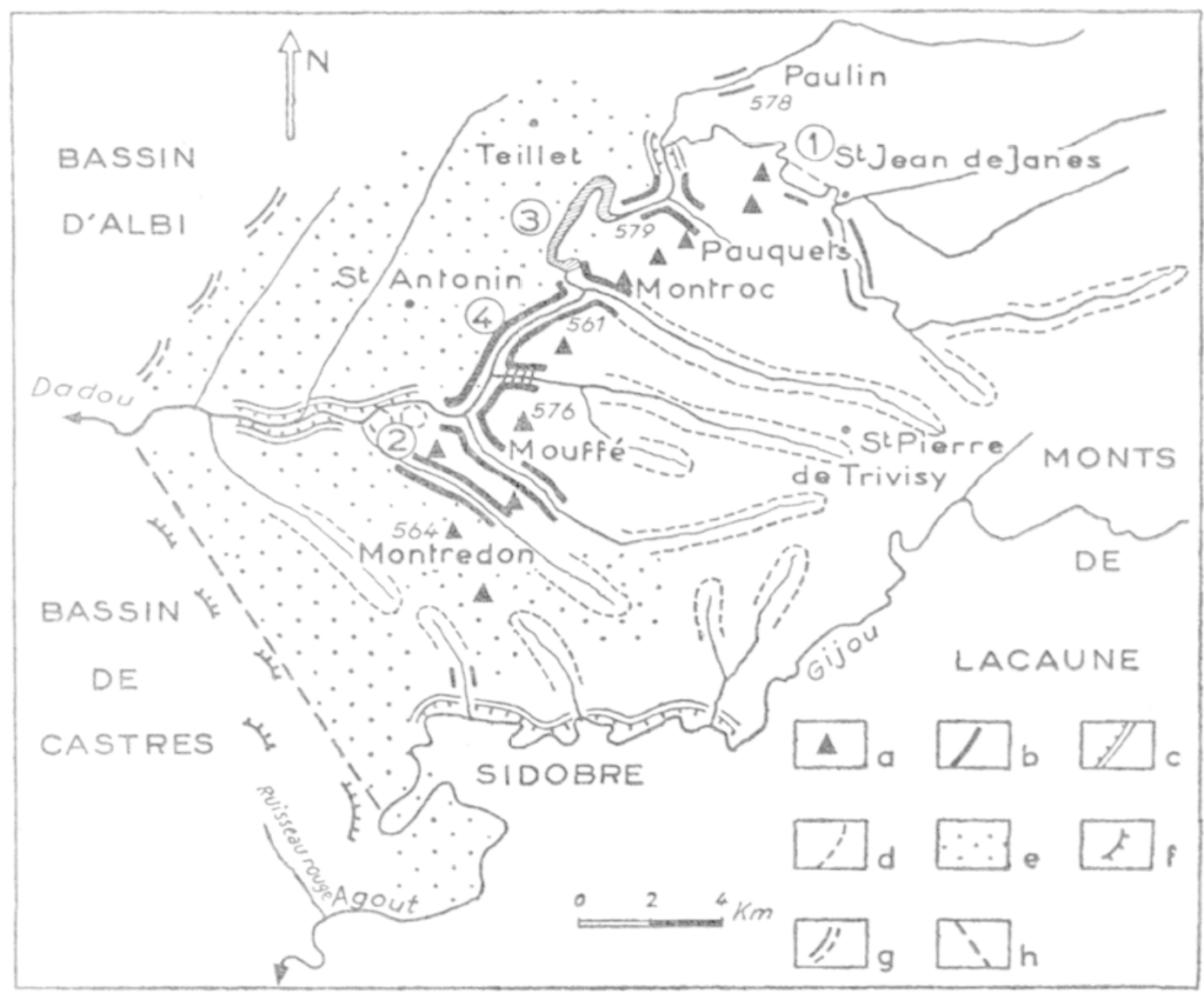

Fig. 4

Les types de vallées.

1. Capture de la basse vallée de l'Ambias.

2. Méandres recoupés de Travanet. ... 3. Barrage artificiel de Rassisse. .. 1. Gorge du Gourp) nigre. - a. Tors. - b. Versants-diaclases des gorses de la muraille de quartz. - c. Versants réglés à rupture de pente sommitale. ... d. lersants convexes, combes solifluees. - e. Argile rouge a gratiers. f. Cucstat des bances de macigno bartoniens. - g. Cuesta du calcaire sammoisien. h. Flexure marginale.

1. I.e matériel quartzeux. --- Le comportement morpholngique de cette roche exceptionnelle n'a pu être établi qu'en comparant lo quartz ch älleurement et le quartz en profondcur grâce aux nombrouses galeries de mino et au tunnel d amenée d eau du barrage de Rassisse. 


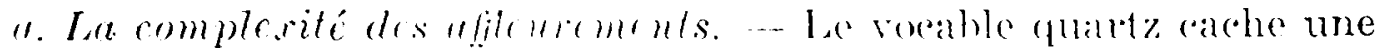
grande variéte de roches nayant entre elles qu un trait commun : la riohesse en silice.

Dans les multiples fractures du soclo siluro-cambrien se somt injectées des solutions minéralisantes an genónal siliconses. Elles on: donné naissanec à des filons derguelefues millimètres à 50 em et plus de barege. Presque tous recoupent la schistosité les protits filons in torieurs à $10 \mathrm{~cm} n$ ont pas de diections prétérentiolles. Ils constituent un réseau romplexe aux mailles serrés (on no voit guipe de coupe

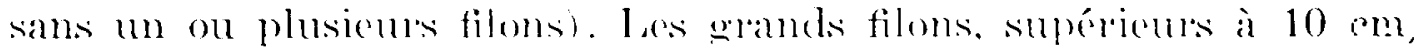

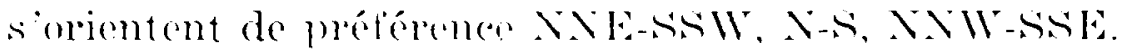

loorigine filonienne n est pas applicable aux grands afleurements de la muraille de quartz proprement dite. On troure de grandes nappes d une centaine de motres d'épaisseur (châtean de Montredon) ef des intumescenees en forme de dome on de champignon (Montroe). Le rapport ave les sehistes nest pas tres net car la stratielatthie est bromillé par la schistosite. L origine reste obseme. L hypothese la plus plansible est edelle de eoulées voloaniques de type rhyolite interstratifiés dans les schistes du Potselamion (16). ('es laves

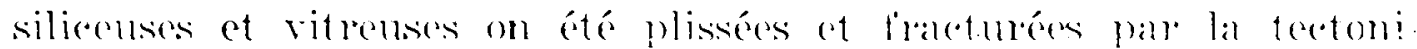
que hereynieme et les mourements posterieurs. Ainsi sexpliquent les decrochements entre les differents alflenurments et la rariabilité des pendages qui, eepondant, demenerent tomjours fouts de loordre

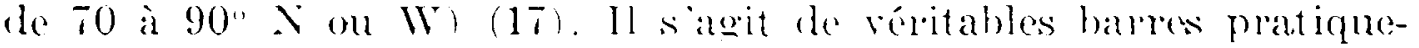
ment indestructibles.

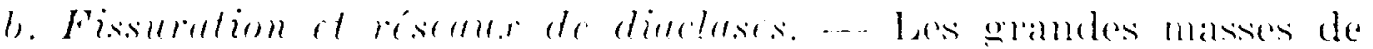
quarty sont immunisés contre la dissolution chimique. Ia mobilisation des silieates, quoique apparente, demeure secondaire. Ies eaux eomantes ne peurent pas attaquer un bloc homogene (aucume trace dattague protonde mais simplement de lasure superficielle, pas de marmites torrentielles par exemple). L'érosion mécanique est impuissante si la roche n'est pas fracturéc.

La diaclase est lólément majeur de la mopholowie du quartz. On on distinoue denx types. Les erandes nappes de pholites sont recentpés par un rósean orthegonal de macro-diachases de $5 \mathrm{~mm}$ à 40 ena de large qui sont espacées de $80 \mathrm{~cm}$ à $2 \mathrm{~m}$. Elles déterminent de gros blocs parallélépipédiques qui constituent l'ólément de base du relief́. Les macro-diaclases sont préformées dans la masse des rhyolites et

(16) P. TEnsrer, Sur un gisement d'alunite au contaci de rhyolites anciens près do Réalmont. Bull. Soc. géol. de France, t. VIII, 1908 , p. 269.

(17) Gisglopp (19). 


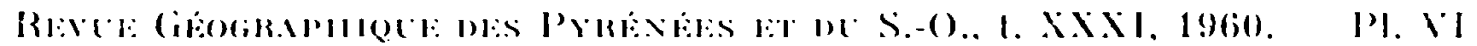

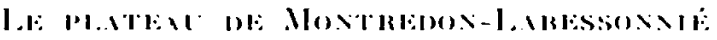

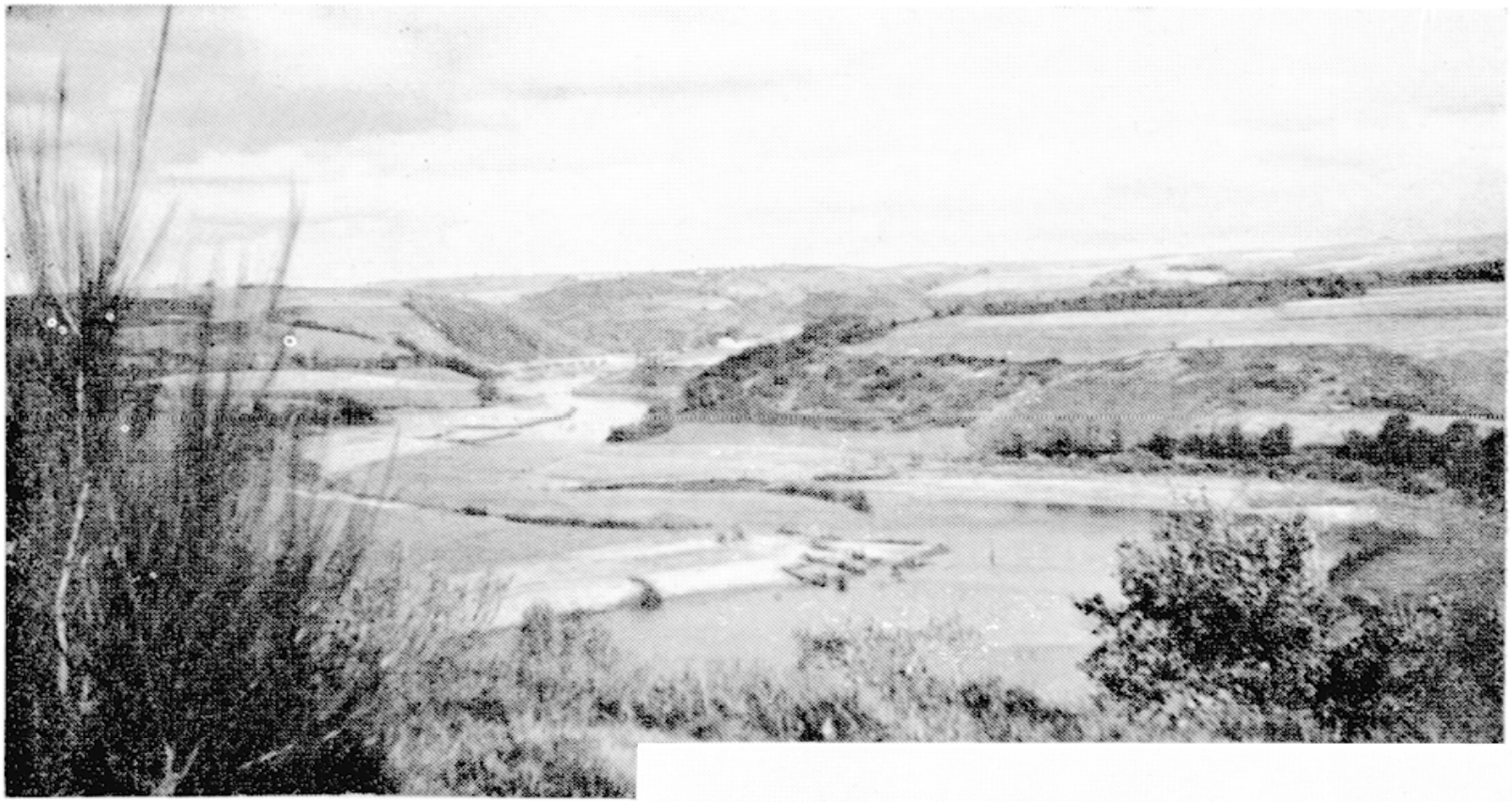

1. I.e platean et la surface des alegiles ronges late de liarratse de Rassisice sur le Dadout.

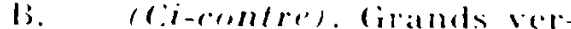
anth a replutere de pente sommita!e (sehistes sericeiteux) daus lat va!lace de lelsout on amont

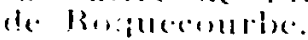
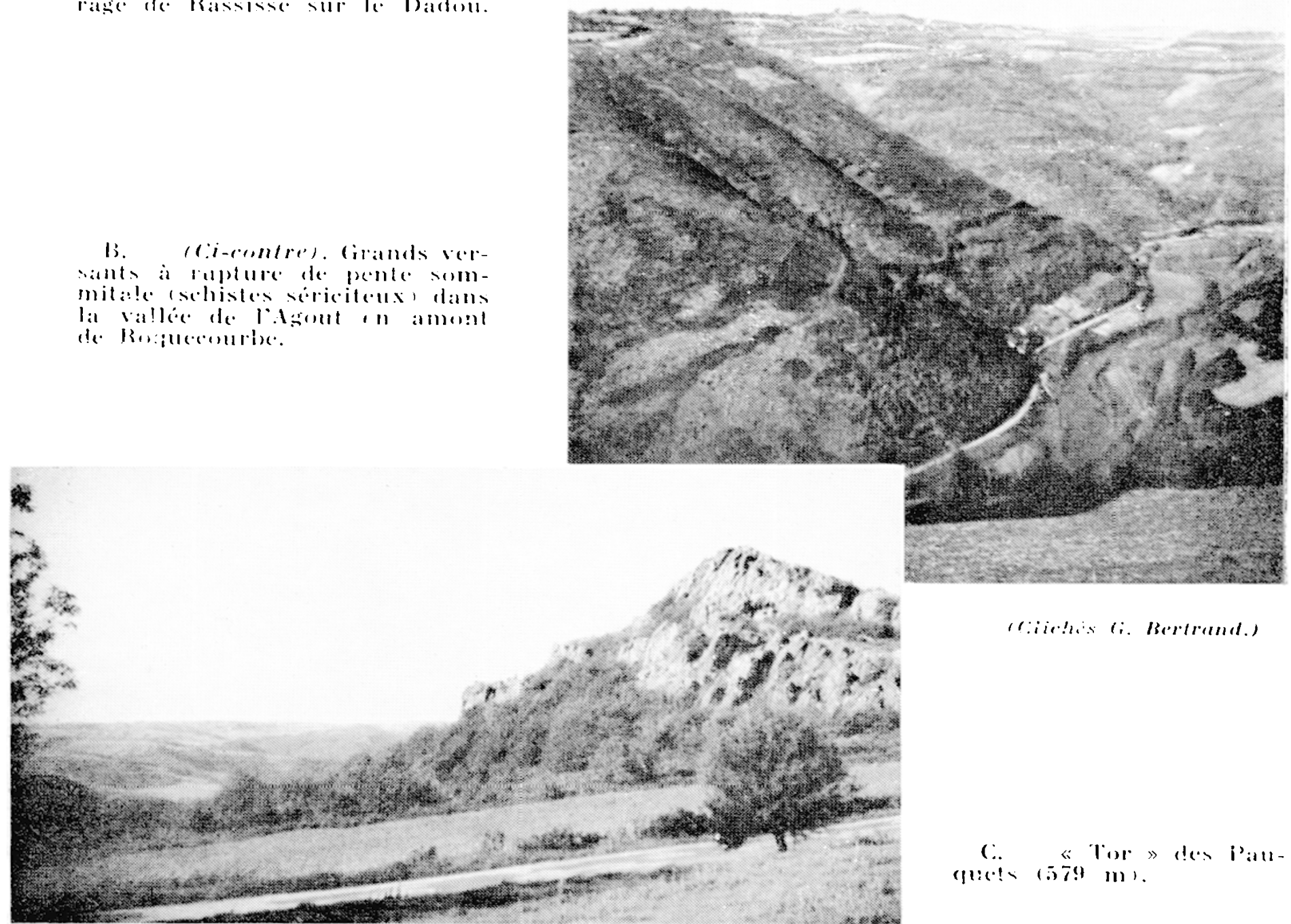

c.. « ror d d . ian(11:0!) $1579 \mathrm{mI})$. 


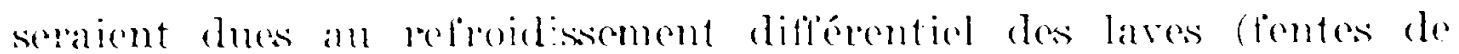
reteat). Dams les filoms, on rencontre surfout des miclo-dialdases a

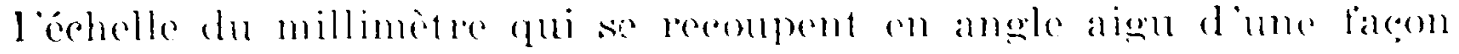

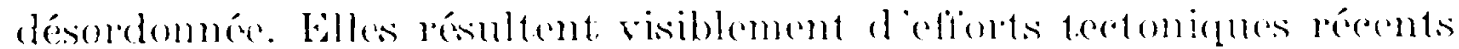

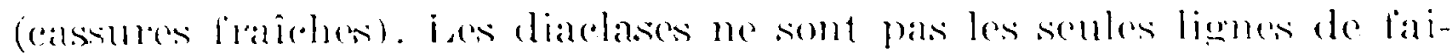
blesse du eomplexe quallyens. Les filons de quatro eollent rarement an toit of an mur. Futre les filons of les epontes, il y a presque tou-

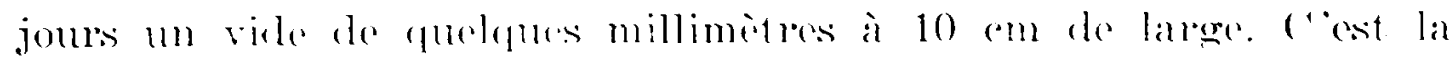

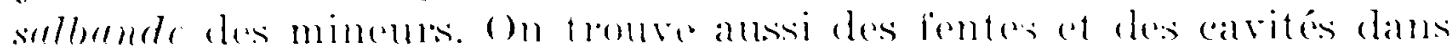
la poche saine à phus de $50 \mathrm{~m}$ sous la surface du sol. Elles ne sont pas

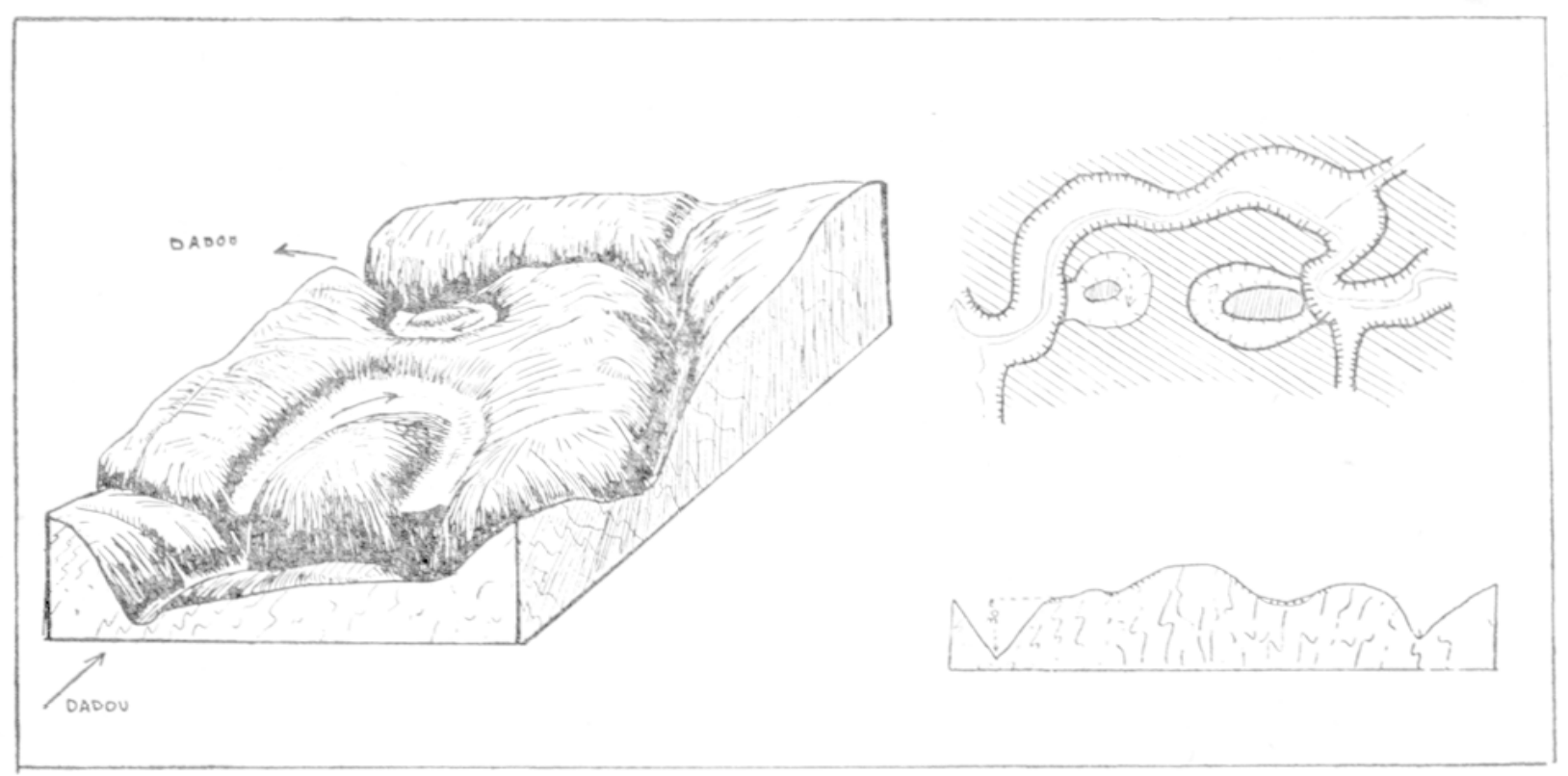

Fi

Les méandres recoupés du badou à Travanet.

dues a lórosion, mais peut atre a des aceidonts dams le métamorphisme (migrations de minéraux) (ls).

L érovion est enudéc par ces zones de faiblesse. I a miero-fissuration facilite la gelivation; lean y eireule librement et travaille is desceller les blocs. I importance morphologique de ces liones d'altérabilité varie suirant les tryes de quartz et leur mode de gisement.

2. Le filon de quartz isolé dans les schistes. - Le problème est de saroir si le quartz renforee on affaiblit la roche encaissante. La silice injectée lit par lit (schistes siliceux) fait corps avec la roche et accroît la résistance à l'érosion chimique et mécanique (soudure

(18) Llopis ILAEO (21). 
des feuillets de schistes). Au rontraire, les nodules d'exsudation font éclater la schistosité et facilitent la pénétration de liane cola entraîne un pourrissement accéléré des schistes voisins.

ice roble des filoms proprement dit est plus amplexe. On reneontre surtout des filons de 1 à $20 \mathrm{~cm}$ de larer. Les filons sont de fôkes colomnes rigides noxées dans des schistes plastiques que hrise ke moindre efïort tectonique. Prescune tous sont diarelasés et beaucoup sont mylonitisés (breches de laille à strueture saceharoüde). Fu général. les filons sont done des zones d'altérabilite par où softectue lattarue des roches meaissantes. La plupart des filons s'entourent d'une aureole de pourriture. Les schistes domnent ume houillie hi-

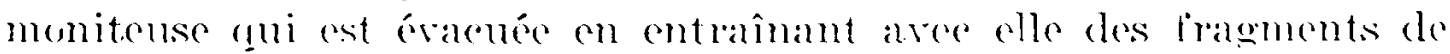

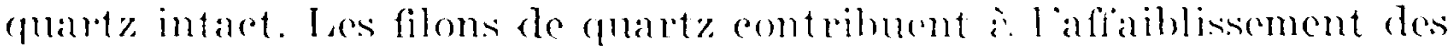
schistes. or qui explique la localisation de boaucoup de petits eours d eaur en fonction des filons de quartz. I autres filons ont été ressoudés par un riment d hématite tres resistant qui noie les diaclases

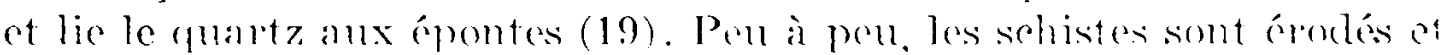
In filon demente on sallie dyte de Roquemante. Moulin du Roi).

3. La muraille de quartz proprement dite. . (" liéteroctite de roches silienuses. On y renemtre tens les types de

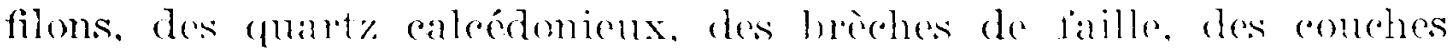

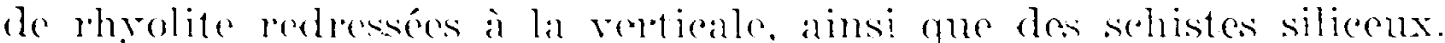

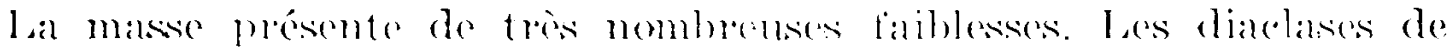
toutes tallles sy recompent of les filoms semt entoures do sallameses. Partois. les épontes sont videns do lour remplissage filonien. Enfin. il y a des sohistes tendres pincés entre les coulées de thyolite. lne eome horizontale relevée le long diune aalerie do mine pres de

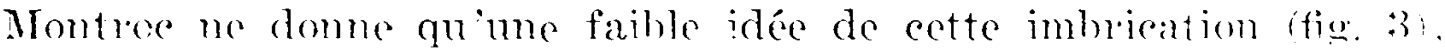
que confirmo le parcours de la galerie d'amenée d'ean de Rassisse.

a. Lu muraille de quertz. zonc d'altérabilité. - I'cau s'infiltur librement entre les roches. Malgré les apparences, la muraille de quartz doit être considéce comme une zone d'intense altérabilité. Elle est minée en profondeur par des eaux d infiltration qui desecndent $̇$ plus de $50 \mathrm{~m}$ sous la surface du sol et qui preparent aetire-

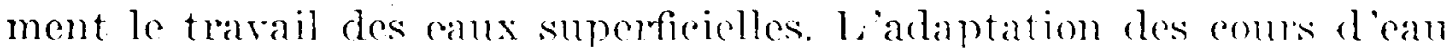
au môle de quartz s'explique facilement. Rnche en soi inaltélahle. le quartz est haché de lignes de faiblesse que los pirières ont utilisées au cours de leur enfoncement. Il sest surtout aci d'un travil do déblaiement : épontes débarrassées de leur remplissage quartzcux.

(19) ASTRE (17). 
nettorage des dóbrin gélivés et des bèdues de faille. Ine fois fixées dans los fisimers. les and comantes nont pu ni changel de place ni beaucoun blareir lem lit surtout si lon tiont eompte de la rapidite

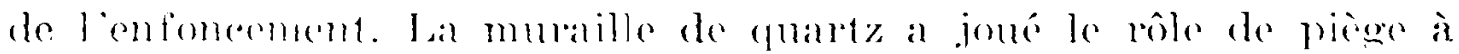

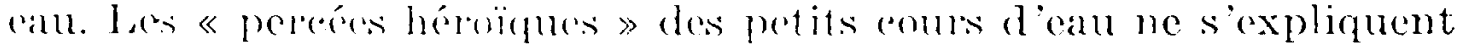
pas antrenont. Its traversent en cluse denormes masses de quartz.

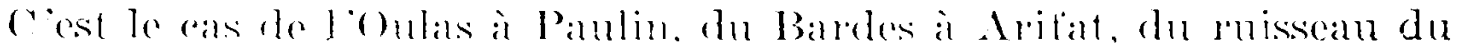
Joulin du lioi de. La's rivieres nont pas mordu dans la roche vive commo olles on domnent limpression, mais elles sont étroitemont adaptés à des lienes de fablelesse entre denx masses de quartz. Ise

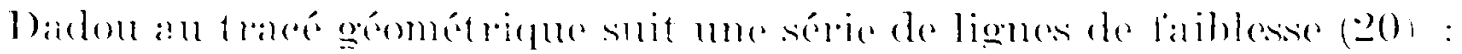

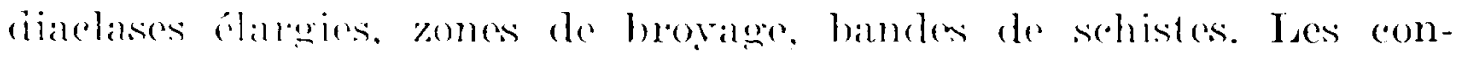

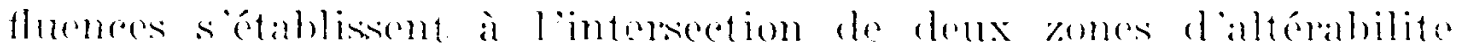

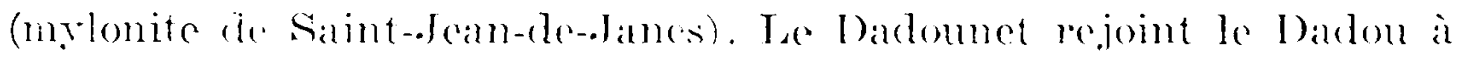

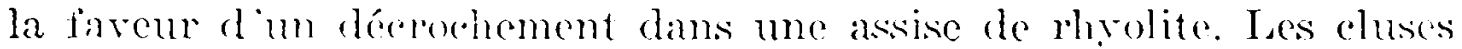
de Panlin et d'dribat paraissont déterminées par drs diaclases.

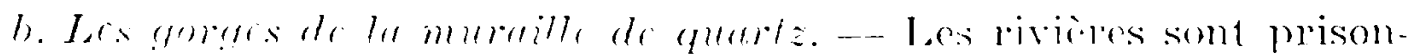
nirjes de la trame filonienne et les valless pestent ralquées sur la

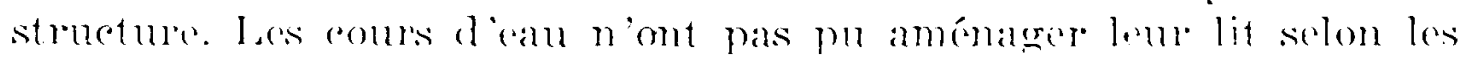
lois de la dynamique fluviale. Si l'om mot a part lo méandre de

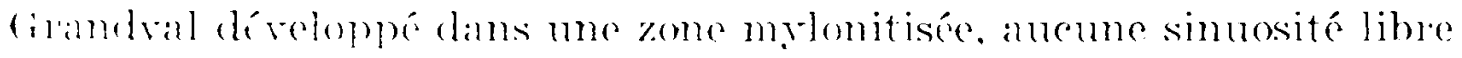

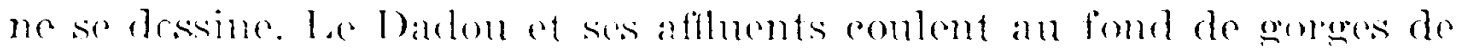

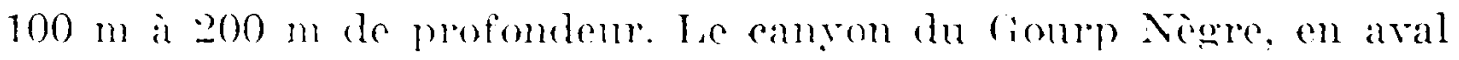
du barage de Rassisse, mesure à peine 10 à 20 m de larere.

la diaclase au sens laloge du terme constitue l élément fondamental du relief. Les resants sont exactement déterminés par les plans de diaclases. Il y a des porte-ì-faux et des eneorbellements dus au hisard du recoupement des fissures. Dans le eanyon du Bardes, le rersant conserve l'inclinaison des diaclases et ne paraît éroluer que par déeollement de bloes. On peut parler de « versant-diaclase » et dans une ecrtaine mesure de « eanyon-diaclase ».

Lorsque la diaclase dispamaît. le quartz est immunisé. Telle est. l'origine des importantes ruptures de pente du profil longitudinal des rivieres. I a muraille de quartzest la zone des rapides et des caseades. Près d'Arifat. le Bardes franchit en caseade une dénivol-

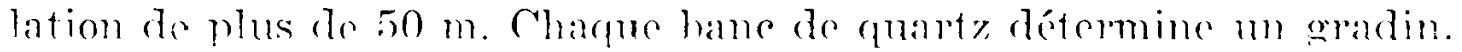
Te fond des ranlées cst oceupé par des amas de bloes en céméral en place analngues anx companés on riviònes de rochers du Sidobre roisin (21).

(211) Inivis (1S).

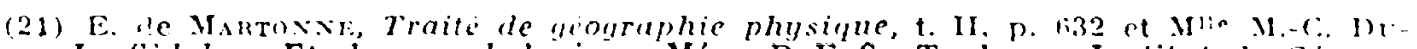
navo, le Silobre. Fitude morphologique. Mém. D. E. S., Toulouse. Institut de Géngraphie, 1960 (ractyl.). 
Le quarty n'oerupe parfois qu'm eoté dr la vallée qui derient

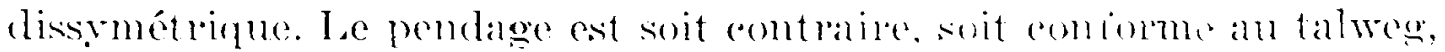
er qui dome beancoup de variete à ces sontes de vallées monoclinales.

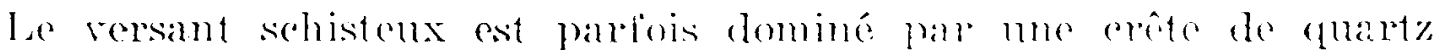

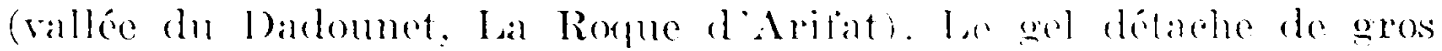

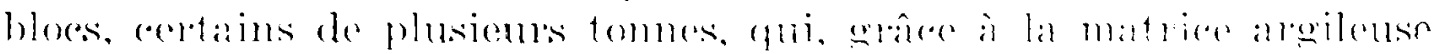
fournic par les schistes solithent sur de putites distances. His forment dans lo has des pontes des chade: de solifurtion (20) qui rappellent par lene forme les amas de hloes qui eomermment les eroupes.

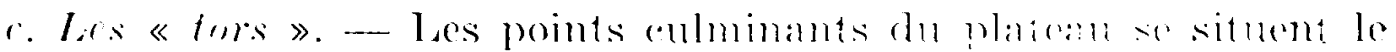
lome de la muraille de quartz. (esont. du Xan s : la hutte du chat-

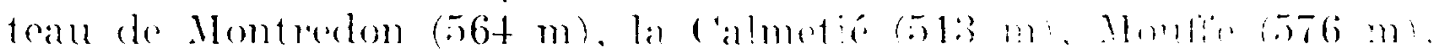

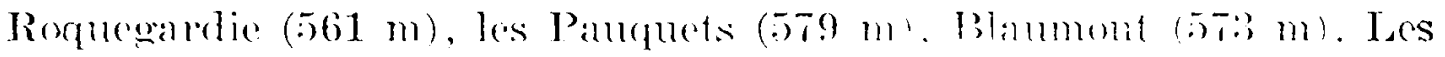

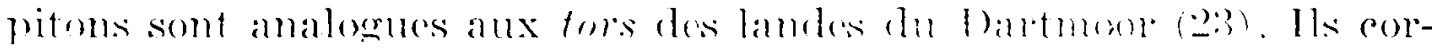

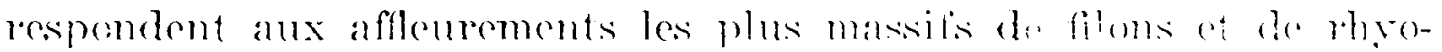
lites. Ides assises silirenses subrerticales ont ete mises on sallie par

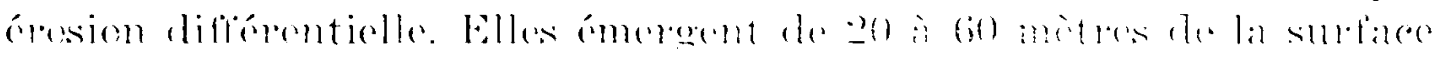

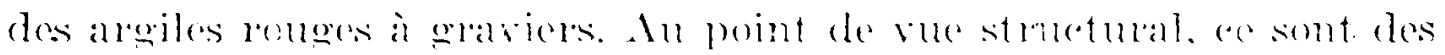

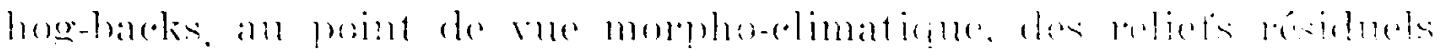

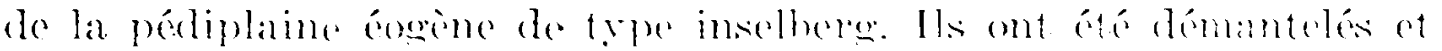

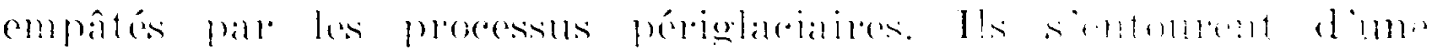

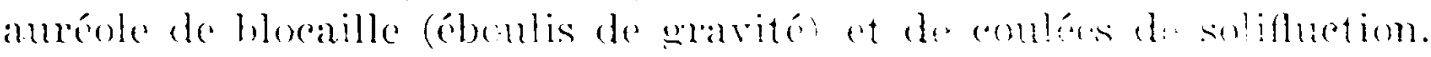

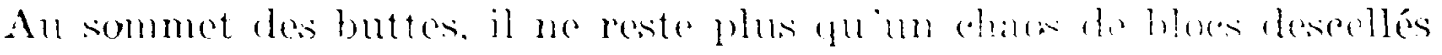

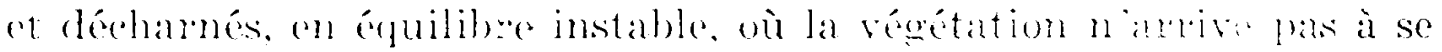
fi:cr.

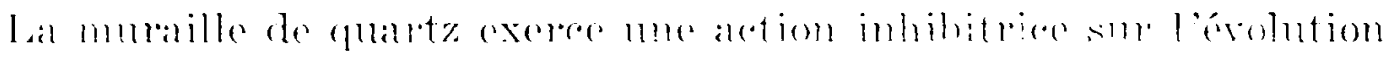

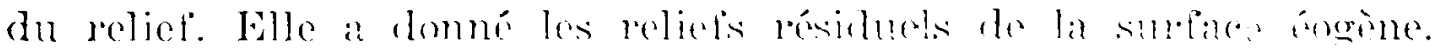

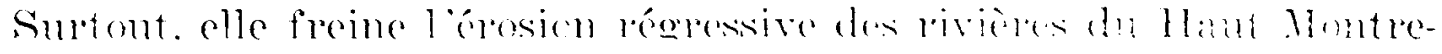
domanis qui se présente $u$ peu à la manière d lun « platon harré »

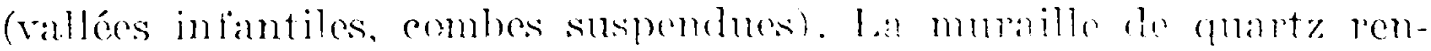
foree le earactere récent et inacheré du reliof montrextommais.

\section{Brbmographe sommine}

1. Batro (H1.). 1928. Le Plateat central de la Franre et se horclare mediterranéenne, Paris, Colin.

2. Exiatrent (H.), 1952. Les plateaux et les gorges du Viant. Rev. SÉogr. Pyrénées, t. XXIII.

3. Derrete (M.), 1952. Les caractères différenticls des roches du Sud-Ouest du Massif central. Publ. Inst. Géogr. Clermont-Ferrand. fasc. 6.

(?2) BAECKEROOT (16).

(23) I,ritox (20). 


\section{Fitudes gréologriques d'ensemble.}

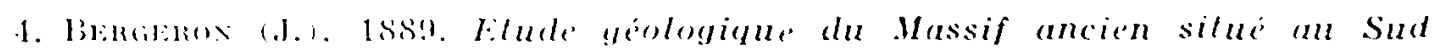
du l'laleall tientral. These sciences, l'aris.

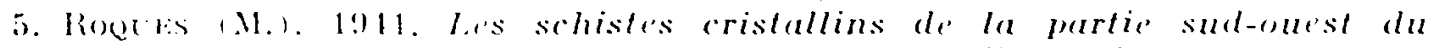
bessif tentral. Thise Seiences, Clemont-ferrand.

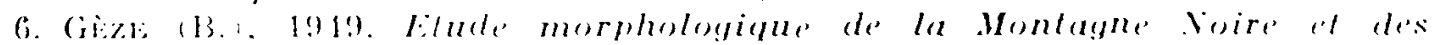
Ciremmes meridionales. lohise seiences. Paris.

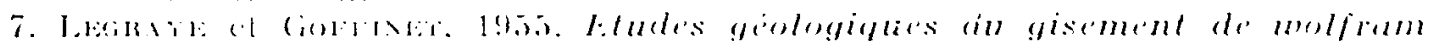

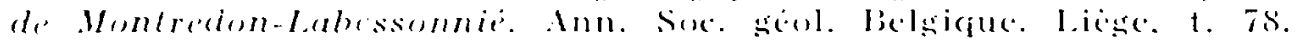

\section{Les argiles rouges à graviers.}

8. Vassigle (6.). 1891. Contribution à létude des terrains tertiaires du Sud-(Muest de la Franee. Bull. Serv. Carte génl. France, n" 19, t. II.

9. MEviden 1..' 1926. Résultats de sondages dans le bassin de castres.

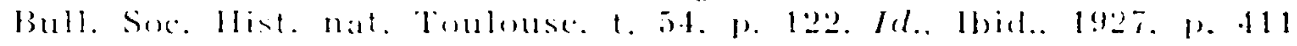
(') 19.45 .216$.

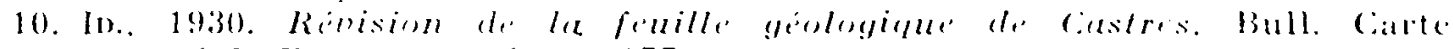
giol. Firance. 1. 34, n" 177 .

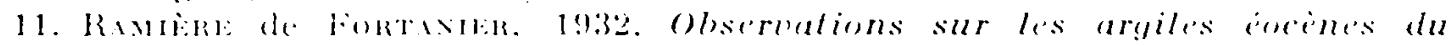
Gastrats. Bull. Sue Hist. nat. Toulumse, t. (i.t. p. th.

12. Vara 1.1. 1945. Etude minéralogique des proninces distributrices de:

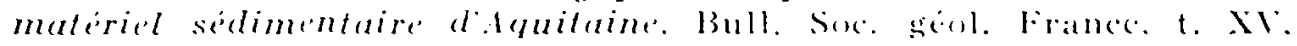
1). 658.

13. In.. 194s. La sedimentation detritique on Aquitaine aux temps tertiair.s. Bull. Soce géol. France, t. Xilli, p. 48.

\section{Modeló périglaciaire.}

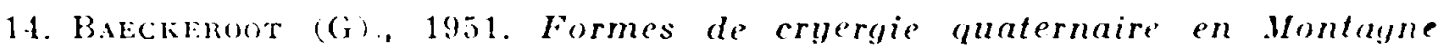
Vuire occidentale. Rev. géser. Pyénés, t. XXII, fase. 2 et 3.

15. H1.. 1952. Le' rile des actions cryonibales quaternaires dans la forme

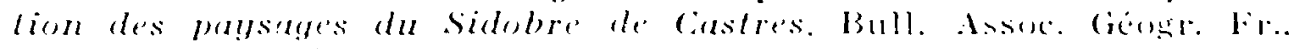
I1" 206. 1. 121-125.

16. In., 1957, Le systeme niehe-coulde et son role dans la formation éu modsti. Bull. Assoce (iensr. Fr.. n" 265. l). 11.

\section{Muraille de quartz.}

17. Astre (G.), 1930. Structure de's breche's quartzeuses filoniennes des environs du Sidobre. Bull. Soce. Hist. nat. Toulduse. 1. is! fasc. 1. I. 167.

18. RMvis, 1939. Roches ef filons d' l'eyrebrune. Bull. Soc. Hist. nat. 'TouIollse. t. 73. P. 127.

19. Gavalutr, 1948. Etude minéralogique et métallogénique de' l'Albigeois cristallin. Ibid.. t. 83.

20. Lexou (1).-L.), 1955. The problem of tors. Geogr. Journal, vol. CXXI. part 4.

21. Leopis L.ano (X.-Y.). Foxtuoté (J.-M.), 1957-1958. Estudio geologico de

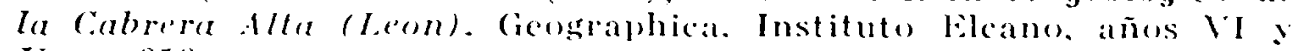
V. 1. 858 .

Cartes. Cartes toposraphiques au $50000^{\circ}$ en hachures Castres et Réalmont

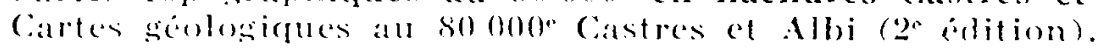

Photographic's a'riense's I.G. N., Missions Lavaur-Castres, Rcialmont, Gaillac-licialmont, et Lacaune-Bédarieux, 1948. 